\title{
Guaranteed cost control of exponential function projective synchronization of delayed complex dynamical networks with hybrid uncertainties asymmetric coupling delays
}

\author{
Wajaree Weera ${ }^{\mathrm{a}}$, Thongchai Botmart ${ }^{\mathrm{b}, *}$, Piyapong Niamsup ${ }^{\mathrm{c}}$, Narongsak Yotha ${ }^{\mathrm{d}}$ \\ ${ }^{a}$ Department of Mathematics, University of Pha Yao, Pha Yao 56000, Thailand. \\ ${ }^{b}$ Department of Mathematics, Khon Kaen University, Khon Kaen 40002, Thailand. \\ ${ }^{c}$ Department of Mathematics, Faculty of Science, Chiang Mai University, Chiang Mai 50200, Thailand. \\ ${ }^{d}$ Department of Applied Mathematics and Statistics, Rajamangala University of Technology Isan, Nakhon Ratchasima 30000, Thailand.
}

Communicated by R. Saadati

\begin{abstract}
The problem of guaranteed cost control for exponential function projective synchronization (EFPS) for complex dynamical networks with mixed time-varying delays and hybrid uncertainties asymmetric coupling delays, composing of state coupling, time-varying delay coupling, and distributed time-varying delay coupling, is investigated. In this work, the uncertainties coupling configuration matrix need not be symmetric or irreducible. The guaranteed cost control for EFPS of delayed complex dynamical networks is considered via hybrid control with nonlinear and mixed linear feedback controls, including error linear term, time-varying delay error linear term, and distributed time-varying delay error linear term. Based on the construction of improved Lyapunov-Krasovskii functional with the technique of dealing with some integral terms, the new sufficient conditions for the existence of the optimal guaranteed cost control laws are presented in terms of linear matrix inequalities (LMIs). The obtained LMIs can be efficiently solved by standard convex optimization algorithms. Moreover, numerical examples are given to demonstrate the effectiveness of proposed guaranteed cost control for EFPS. The results in this article generalize and improve the corresponding results of the recent works.
\end{abstract}

Keywords: Guaranteed cost control, exponential function projective synchronization, complex dynamical networks, hybrid uncertainties asymmetric coupling.

2010 MSC: 37N35, 37C75, 93B52.

(C)2018 All rights reserved.

\section{Introduction}

Complex dynamical networks, as an interesting subject, have been thoroughly investigated for the past decades. The theories and applications of complex dynamical networks have become a hot topic in the breakthrough of research methods of complex dynamical networks. These networks exhibit very

\footnotetext{
*Corresponding author

Email addresses: wajaree.we@up.ac.th (Wajaree Weera), thongbo@kku.ac.th (Thongchai Botmart), piyapong.n@cmu.ac.th (Piyapong Niamsup), narongsak. yo@rmuti.ac.th (Narongsak Yotha)
}

doi: $10.22436 /$ jnsa.011.04.11

Received: 2017-09-12 Revised: 2018-01-10 Accepted: 2018-01-11 
complicated behavior and can be used to model and explain many complex systems in nature such as computer networks, the world wide web, cellular and metabolic networks, transportation networks, communication networks, disease transmission networks, electrical power grids $[2,12,16,34,36,37]$ and so forth.

The problem of synchronization in complex dynamical networks (CDNs) has been extensively investigated over the part few decades. Synchronization of CDNs is one of the most important dynamical mechanisms for creating order in CDNs. Meanwhile, a number of methods have been developed for the synchronization of CDNs including complete synchronization (CS) $[6,22,41]$, generalized synchronization (GS) [38], exponential synchronization (ES) [4, 42], projection synchronization (PS) [24, 44], etc.. Recently, the new type of synchronization phenomenon in CDNs, called function projection synchronization (FPS) was introduced [1, 10,11,31,44]. In these works, the nodes of CDNs could be synchronize up to an equilibrium point or periodic orbit with a desired scaling function. In [44], the FPS in driveresponse dynamical networks with coupled partially linear chaotic systems was presented in which the identical node dynamics was assumed and a simple control law was used. In [10], the problem of FPS of (CDNs) with or without external disturbances using error feedback control and adaptive error feedback control was investigated. In [11], a hybrid feedback control method was proposed for achieving FPS in CDNs with constant time delay and time-varying coupling delay. Function projective synchronization for complex networks with asymmetric coupling matrix via adaptive and pinning feedback control was considered in [31]. However, all their results was concerned with FPS of CDNs. As far as we know, there have still been few studies on exponential function projective synchronization (EFPS) for CDNs. So, it is challenging to deal with the EFPS problem of CDNs with mixed time-varying delays and hybrid uncertainties asymmetric coupling delays.

In the past few decades, control problems for synchronization have been widely studied in CDNs. Many important synchronization control methods have also been developed for CDNs, for instance, feedback control [10, 11], intermittent control [4,5], sampled-data control [30], nonlinear feedback control $[9,32]$, pinning control [14, 29, 33, 43], adaptive control [25, 31, 40, 45], pinning adaptive control $[19,25,31,39]$, and other control methods. When controlling a real plant, it is always desirable to design a control system not only asymptotical stability but also guaranteed adequate level of performance. One of the design approaches for solving this problem is the so-called guaranteed cost control approach first introduced by Chang and Peng [7]. The guaranteed cost control for various dynamic systems has been investigated in $[3,8,21,26-28,35]$. In [18], the guaranteed cost synchronization for a complex dynamical network via dynamic feedback control was proposed. In [17], synchronization problem of a complex dynamical network with probabilistic switches for the topology of a complex network was investigated. Decentralized guaranteed cost dynamic feedback controller is designed to achieve the synchronization of the network. The guaranteed cost control of synchronization for a class of complex delayed dynamical networks with uncertain inner coupling configuration was investigated [20]. The coupling delay and node delay was considered in the networks. In [23], the guaranteed cost synchronization control problem of some general complex dynamical networks with delay was investigated and dynamic feedback controller were designed for the pinning control of parts of the nodes in order for the system to achieve the guaranteed cost synchronization. Moreover, the guaranteed cost synchronization of delayed complex dynamical networks with node uncertainties and coupling uncertainties was presented in [15]. So far, unfortunately, there have still been few papers related to the topic of guaranteed cost synchronization of a CDNs with mixed time-varying delays in the dynamical nodes and in the hybrid uncertainties coupling, including constant coupling, discrete time-varying delay coupling, and distributed time-varying delay coupling, simultaneously. Therefore, it is challenging to solve this guaranteed cost synchronization problem for CDNs.

Motivated by the above discussions, this work is one of the first reports of such investigation to further develop the guaranteed cost control problem for EFPS of complex dynamical networks with mixed time-varying delays and hybrid uncertainties asymmetric coupling delays. The main contributions of our study are as the following. Firstly, the definition of exponential function projective synchronization for 
complex dynamical networks is introduced. Secondly, the considered time-varying delays are different from the time-delay case in [15]. The time-varying delays were mixed between discrete and distributed time-varying delays in the dynamical nodes and in hybrid uncertainties asymmetric coupling, simultaneously. Thirdly, we do not assume that the uncertainties coupling configuration matrix needs not be symmetric or irreducible, which is different from coupling in Refs. [17, 20]. Finally, for the control method, the guaranteed cost control problem for EFPS is studied via hybrid control with nonlinear and mixed linear feedback control, containing error linear term, time-varying delay error linear term, and distributed time-varying delay error linear term. The EFPS is different from control method in $[17,18,23]$. By constructing new and improved Lyapunov-Krasovskii functional with the technique of dealing with some integral terms, the new sufficient conditions for existence of the guaranteed cost control via hybrid control are expressed as linear matrix inequalities (LMIs). The obtained LMIs can be efficiently solved by standard convex optimization algorithms. Numerical examples are included to show the effectiveness of the proposed hybrid control scheme.

The rest of the paper is organized as follows. Section 2 provides some mathematical preliminaries and network model. Section 3 presents EFPS of complex dynamical network with mixed time-varying delays and hybrid uncertainties asymmetric coupling by hybrid control. Numerical examples are given in Section 4. Finally, the conclusions are provided in Section 5.

\section{Network model and mathematic preliminaries}

Consider a complex dynamical network consisting of $N$ identical coupled nodes, with each node being an n-dimensional nonlinear dynamical system

$$
\begin{aligned}
\dot{x}_{i}(t)= & f\left(t, x_{i}(t), x_{i}\left(t-\tau_{1}(t)\right), \int_{t-\sigma_{1}(t)}^{t} x_{i}(s) d s\right)+\rho_{1} \sum_{j=1}^{N} w_{i j}^{1}\left(G_{1}+\Delta G_{1}(t)\right) x_{j}(t) \\
& +\rho_{2} \sum_{j=1}^{N} w_{i j}^{2}\left(G_{2}+\Delta G_{2}(t)\right) x_{j}\left(t-\tau_{2}(t)\right)+\rho_{3} \sum_{j=1}^{N} w_{i j}^{3}\left(G_{3}+\Delta G_{3}(t)\right) \int_{t-\sigma_{2}(t)}^{t} x_{j}(s) d s \\
& +U_{i}(t), \quad t \geqslant 0, i=1,2, \ldots, N, \\
x_{i}(t)= & \phi_{i}(t), \quad t \in\left[-\tau_{\max }, 0\right], \quad \tau_{\max }=\max \left\{\tau_{1}, \tau_{2}, \tau_{3}, \sigma_{1}, \sigma_{2}, \sigma_{3}\right\},
\end{aligned}
$$

where $x_{i}(t)=\left(x_{i 1}(t), x_{i 2}(t), \ldots, x_{i n}(t)\right)^{\top} \in \mathbb{R}^{n}$ is the state vector of $i$ th node; $f: \mathbb{R}_{+}^{n} \times \mathbb{R}^{n} \times \mathbb{R}^{n} \times \mathbb{R}^{n} \rightarrow \mathbb{R}^{n}$ is a smooth nonlinear vector function which describes the local dynamics of nodes, and is continuously differentiable and capable of performing abundant dynamical behaviors such as equilibrium points, periodic orbits, and chaos; $\mathcal{U}_{\mathfrak{i}}(t) \in \mathbb{R}^{\mathrm{m}}$ is the control input of the node $i$; the constants $\rho_{1}, \rho_{2}, \rho_{3}>0$ denote the non-delayed and delayed coupling strength, respectively; $G_{1}, G_{2}, G_{3} \in \mathbb{R}^{n \times n}$ are constant inner-coupling matrices; $\Delta \mathrm{G}_{1}(\mathrm{t}), \Delta \mathrm{G}_{2}(\mathrm{t}), \Delta \mathrm{G}_{3}(\mathrm{t}) \in \mathbb{R}^{\mathrm{n} \times \mathrm{n}}$ are the uncertainties of inner coupling matrices; $W_{1}=\left(w_{i j}^{1}\right)_{N \times N}, W_{2}=\left(w_{i j}^{2}\right)_{N \times N}, W_{3}=\left(w_{i j}^{3}\right)_{N \times N} \in \mathbb{R}^{N \times N}$ are the coupling configuration matrices representing the coupling weights and topological structure for non-delayed configuration and delayed one at time $t$, respectively, in which $w_{i j}^{1}, w_{i j}^{2}$ and $w_{i j}^{3}$ are defined as follows: if there is a connection between node $i$ and node $j(j \neq i)$, then $w_{i j}^{1}>0, w_{i j}^{2}>0, w_{i j}^{3}>0$; otherwise, $w_{i j}^{1}=0, w_{i j}^{2}=0, w_{i j}^{3}=0(j \neq i)$, and the diagonal elements of matrices $W_{1}, W_{2}$, and $W_{3}$ are defined by

$$
w_{i i}^{1}=-\sum_{j=1, i \neq j}^{N} w_{i j}^{1}, w_{i i}^{2}=-\sum_{j=1, i \neq j}^{N} w_{i j}^{2}, w 3_{i i}=-\sum_{j=1, i \neq j}^{N} w_{i j}^{3}, i=1,2, \ldots, N .
$$

Suppose that $C\left(\left[-\tau_{\max }, 0\right], \mathcal{R}^{\mathfrak{n}}\right)$ is the Banach space of continuous functions with the norm

$$
\left\|\phi_{i}\right\|=\sup _{-\tau_{\max } \leqslant s \leqslant 0}\left\|\phi_{i}(s)\right\| .
$$

The initial condition function $\phi_{i}(t)$ denotes a continuous vector-valued initial function of $t \in\left[-\tau_{\max }, 0\right]$. Under the initial conditions, we always assume that (2.1) has a unique solution. 
For this synchronization scheme, we set the error vector in the form $e_{i}(t)=x_{i}(t)-\alpha(t) s(t), i=$ $1, \ldots, N$, where $\alpha(t)$ is a continuously bounded differentiable function. Then, substituting it into (2.1), the error dynamics network is given to

$$
\begin{aligned}
\dot{e}_{i}(t)= & \dot{x}_{i}(t)-\dot{\alpha}(t) s(t)-\alpha(t) \dot{s}(t) \\
= & f\left(x_{i}(t), x_{i}\left(t-\tau_{1}(t)\right), \int_{t-\sigma_{1}(t)}^{t} x_{i}(s) d s\right)-\alpha(t) f\left(s(t), s\left(t-\tau_{1}(t)\right), \int_{t-\sigma_{1}(t)}^{t} s(\theta) d \theta\right) \\
& +\rho_{1} \sum_{j=1}^{N} w_{i j}^{1}\left(G_{1}+\Delta G_{1}(t)\right) e_{j}(t)+\rho_{2} \sum_{j=1}^{N} w_{i j}^{2}\left(G_{2}+\Delta G_{2}(t)\right) e_{j}\left(t-\tau_{2}(t)\right) \\
& +\rho_{3} \sum_{j=1}^{N} w_{i j}^{3}\left(G_{3}+\Delta G_{3}(t)\right) \int_{t-\sigma_{2}(t)}^{t} e_{j}(s) d s-\dot{\alpha}(t) s(t)+U_{i}(t), \quad i=1, \ldots, N .
\end{aligned}
$$

Definition 2.1. Network (2.1) with mixed time-varying delay is said to achieve exponential function projective synchronization (EFPS) if there exists $M_{i} \geqslant 1, \delta>0$ and a continuously differentiable scaling function $\alpha(t)$ such that

$$
\lim _{t \rightarrow \infty}\left\|e_{i}(t)\right\|=\lim _{t \rightarrow \infty}\left\|x_{i}(t)-\alpha(t) s(t)\right\| \leqslant M_{i}\left\|\phi_{i}-\alpha \omega\right\| e^{-\delta t}, \quad \forall t>0, \quad i=1,2, \ldots, N,
$$

where $\|$.$\| stands for the Euclidean vector norm and s(t) \in R^{n}$ can be either an equilibrium point, or a (quasi-)periodic orbit, or an orbit of a chaotic attractor, which satisfies

$$
\dot{s}(t)=f\left(s(t), s\left(t-\tau_{1}(t)\right), \int_{t-\sigma_{1}(t)}^{t} s(\theta) d \theta\right), \quad \forall t>0, \quad s(t)=\omega(t), \quad t \in\left[-\tau_{\max }, 0\right] .
$$

Remark 2.2. If the scaling function $\alpha(t)=1$ or $\alpha(t)=-1$, then the synchronization problem will be reduced to the complete synchronization or anti-synchronization. If the scaling function $\alpha(t)=0$, then the synchronization problem will be turned into a chaos control problem.

In order to stabilize the error system of uncertain delayed complex dynamical network (2.2), the following dynamic hybrid controllers $\mathfrak{U}_{\mathfrak{i}}(t)$ such as

$$
\mathcal{U}_{i}(t)=U_{i 1}(t)+U_{i 2}(t), i=1,2, \ldots, N,
$$

where

$$
\mathcal{u}_{i 1}(t)=\dot{\alpha}(t) s(t), \quad u_{i 2}(t)=B_{1 i} u_{i}(t)+B_{2 i} u_{i}\left(t-\tau_{3}(t)\right)+B_{3 i} \int_{t-\sigma_{3}(t)}^{t} u_{i}(s) d s,
$$

where $u_{i}(t)=K_{i} e_{i}(t)$ and $K_{i}, i=1,2, \ldots, N$, is constant matrix control gain. In this work, our goal is to design suitable $K_{i}$ such that the complex network (2.2) is exponentially stable. Then, substituting it into (2.2), we get the following:

$$
\begin{aligned}
\dot{e}_{i}(t)= & \dot{x}_{i}(t)-\dot{\alpha}(t) s(t)-\alpha(t) \dot{s}(t) \\
= & f\left(x_{i}(t), x_{i}\left(t-\tau_{1}(t)\right), \int_{t-\sigma_{1}(t)}^{t} x_{i}(s) d s\right)-\alpha(t) f\left(s(t), s\left(t-\tau_{1}(t)\right), \int_{t-\sigma_{1}(t)}^{t} s(\theta) d \theta\right) \\
& +\rho_{1} \sum_{j=1}^{N} w_{i j}^{1}\left(G_{1}+\Delta G_{1}(t)\right) e_{j}(t)+\rho_{2} \sum_{j=1}^{N} w_{i j}^{2}\left(G_{2}+\Delta G_{2}(t)\right) e_{j}\left(t-\tau_{2}(t)\right) \\
& +\rho_{3} \sum_{j=1}^{N} w_{i j}^{3}\left(G_{3}+\Delta G_{3}(t)\right) \int_{t-\sigma_{2}(t)}^{t} e_{j}(s) d s+B_{1 i} u_{i}(t)+B_{2 i} u_{i}\left(t-\tau_{3}(t)\right), \\
& +B_{3 i} \int_{t-\sigma_{3}(t)}^{t} u_{i}(s) d s, \quad i=1, \ldots, N, \\
e_{i}(t)= & \varphi_{i}(t)=\phi_{i}(t)-\alpha(t) \omega(t), \quad t \in\left[-\tau_{\max }, 0\right], \quad i=1, \ldots, N .
\end{aligned}
$$


Define the following quadratic cost function of the associated network (2.2) as follows:

$$
J=\sum_{i=1}^{N} \int_{0}^{\infty}\left[e_{i}^{\top}(t) Q_{1 i} e_{i}(t)+u_{i}^{\top}(t) Q_{2 i} u_{i}(t)\right] d t
$$

where $\mathrm{Q}_{1 i}, \mathrm{Q}_{2 i} \in \mathbb{R}^{\mathrm{n} \times \mathrm{n}}$ are positive definite matrices. The guaranteed cost control problem to be addressed in this section is formulated as follows.

Definition 2.3. For the uncertain delayed complex dynamical network (2.1) and the quadratic cost function (2.6), if there exist feedback control laws $u_{i}(t)=K_{i} e_{i}(t), i=1,2, \ldots, N$, and a positive number $J^{*}$ such that the error network (2.5) is EFPS, and the corresponding value of cost function (2.6) satisfies $J \leqslant J^{*}$, then $J^{*}$ is said to be a guaranteed cost and $u_{i}(t), i=1,2, \ldots, N$ are said to be the guaranteed cost control laws for the EFPS of delayed complex dynamical network (2.1) with the quadratic cost function (2.6).

Remark 2.4. If $\tau_{1}(t)=\tau_{2}(t)=0, \sigma_{1}(t)=\sigma_{2}(t)=0, \rho_{1}=1, \rho_{2}=\rho_{3}=0$ the network model (2.1) turns into the complex dynamical network proposed by Lee et al. [18] as

$$
\dot{x}_{i}(t)=f\left(x_{i}(t)\right)+\sum_{j=1}^{N} w_{i j}^{1} x_{j}(t)+U_{i}(t), \quad i=1, \ldots, N .
$$

If $\rho_{1}=\rho_{3}=0, \rho_{2}=1$, for constant delays $\tau_{1}(t)=\tau_{1}, \tau_{2}(t)=\tau_{2}$ and $\sigma_{1}(t)=\sigma_{2}(t)=0$, then the network model (2.1) is translated into

$$
\dot{x}_{i}(t)=f\left(x_{i}(t), x_{i}\left(t-\tau_{1}\right)\right)+\sum_{j=1}^{N} w_{i j}^{1}\left(G_{1}+\Delta G_{1}(t)\right) x_{j}\left(t-\tau_{2}\right)+U_{i}(t), \quad i=1, \ldots, N .
$$

The complex dynamical network (2.7) was considered in [20]. For time-varying delays, $\tau_{1}(t) \geqslant 0, \tau_{2}(t) \geqslant 0$ and $f\left(x_{i}(t), x_{i}\left(t-\tau_{1}\right)\right)=(A+\Delta A) x_{i}(t)+g_{1}\left(x_{i}(t)\right)+g_{2}\left(x_{i}\left(t-\tau_{1}(t)\right)\right)$, the network model (2.7) turns into the complex dynamical network presented by He et al. [15]. Hence, our network model (2.1) is more generalized than previous network models, which can be regarded as special cases of the complex dynamical network (2.1).

In the rest of this paper, we need the following assumptions and some lemmas.

Assumption 2.5. The time-varying delays functions $\tau_{\mathfrak{i}}(\mathrm{t})$ and $\sigma_{\mathfrak{i}}(\mathrm{t}), i=1,2,3$ satisfy conditions that $\tau_{\mathfrak{i}}(\mathrm{t})$ is differentiable, $0 \leqslant \tau_{\mathfrak{i}}(t) \leqslant \tau_{\mathfrak{i}}, 0 \leqslant \sigma_{\mathfrak{i}}(t) \leqslant \sigma_{i}, i=1,2,3,0 \leqslant \dot{\tau}_{\mathfrak{i}}(t) \leqslant \beta_{j}<1, j=1,2$ and $\dot{\tau}_{3}(t) \leqslant \beta_{3} \neq 1$.

Assumption 2.6. The matrices $\Delta \mathrm{G}_{1}(\mathrm{t}), \Delta \mathrm{G}_{2}(\mathrm{t}), \Delta \mathrm{G}_{3}(\mathrm{t}) \in \mathbb{R}^{\mathrm{n} \times \mathrm{n}}$ denote the uncertainties in system and they are of the forms

$$
\Delta \mathrm{G}_{1}(\mathrm{t})=\mathrm{D}_{1} \mathrm{~F}(\mathrm{t}) \mathrm{E}_{1}, \quad \Delta \mathrm{G}_{2}(\mathrm{t})=\mathrm{D}_{2} \mathrm{~F}(\mathrm{t}) \mathrm{E}_{2}, \quad \Delta \mathrm{G}_{3}(\mathrm{t})=\mathrm{D}_{3} \mathrm{~F}(\mathrm{t}) \mathrm{E}_{3},
$$

where $\mathrm{D}_{1}, \mathrm{D}_{2}, \mathrm{D}_{3}, \mathrm{E}_{1}, \mathrm{E}_{2}, \mathrm{E}_{3}$ are known constant matrices and $\mathrm{F}(\mathrm{t})$ unknown real time-varying matrices with Lebesgue measurable elements bounded by

$$
F^{\top}(t) F(t) \leqslant I .
$$

Lemma 2.7 ([13, Cauchy inequality]). For any symmetric positive definite matrix $N \in M^{\mathrm{n} \times \mathrm{n}}$ and $x, y \in \mathbb{R}^{\mathrm{n}}$ we have

$$
\pm 2 x^{\top} y \leqslant x^{\top} N x+y^{\top} N^{-1} y .
$$

Lemma 2.8 ([13]). For any constant symmetric matrix $M \in \mathrm{R}^{\mathrm{m} \times \mathrm{m}}, M=M^{\top}>0, \gamma>0$, vector function $\omega:[0, \gamma] \rightarrow \mathbb{R}^{\mathrm{m}}$ such that the integrations concerned are well defined

$$
\left(\int_{0}^{\gamma} \omega^{\top}(s) d s\right)^{\top} M\left(\int_{0}^{\gamma} \omega(s) d s\right) \leqslant \gamma \int_{0}^{\gamma} \omega^{\top}(s) M \omega(s) d s .
$$


Lemma 2.9 ([13], Schur complement). Given constant symmetric matrices $X, Y, Z$ with appropriate dimensions satisfying $X=X^{\top}, Y=Y^{\top}>0$, then $X+Z^{\top} Y^{-1} Z<0$ if and only if

$$
\left(\begin{array}{cc}
X & Z^{\top} \\
Z & -Y
\end{array}\right)<0 \text { or } \quad\left(\begin{array}{cc}
-Y & Z \\
Z^{\top} & X
\end{array}\right)<0
$$

\section{Guaranteed cost EFPS of delayed complex dynamical network}

In this section, we give some sufficient conditions for guaranteed cost EFPS of complex dynamical network with discrete and distributed time-varying delays and hybrid uncertainties asymmetric coupling delays (2.1) via hybrid control. Let us set

$$
\begin{aligned}
\left\|\varphi_{i}\right\|= & \left\|e_{i}(0)\right\|, \quad\left\|\varphi_{i}\right\|_{c l}=\sup _{-\tau_{\max } \leqslant s \leqslant 0}\left\|e_{i}(s)\right\|, \eta_{i}=\lambda_{\min }\left(\mathrm{P}_{i}^{-1}\right), \quad \rho_{i}=\lambda_{\max }\left(\mathrm{P}_{i}^{-1}\right), \\
\sigma_{i}= & \sum_{i=1}^{N}\left[\lambda_{\max }\left(P_{i}^{-1} R_{i} P_{i}^{-1}\right) \frac{1-e^{-2 \delta \tau_{1}}}{2 \delta}+\lambda_{\max }\left(P_{i}^{-1} S_{i} P_{i}^{-1}\right) \frac{1-e^{-2 \delta \tau_{2}}}{2 \delta}\right. \\
& +\lambda_{\max }\left(P_{i}^{-1} Z_{i}^{\top} U_{i}^{-1} Z_{i} P_{i}^{-1}\right) \frac{1-e^{-2 \delta \tau_{3}}}{2 \delta}+\sigma_{1}^{2} \lambda_{\max }\left(P_{i}^{-1} V_{i} P_{i}^{-1}\right) \frac{1-e^{-2 \delta \sigma_{1}}}{2 \delta} \\
& \left.+\sigma_{2}^{2} \lambda_{\max }\left(P_{i}^{-1} \mathrm{~T}_{i} P_{i}^{-1}\right) \frac{1-e^{-2 \delta \sigma_{2}}}{2 \delta}+\sigma_{3}^{2} \lambda_{\max }\left(P_{i}^{-1} Z_{i}^{\top} W_{i}^{-1} Z_{i} P_{i}^{-1}\right) \frac{1-e^{-2 \delta \sigma_{3}}}{2 \delta}\right], \\
\mathcal{M}_{i}= & \rho_{i}\left\|\varphi_{i}\right\|^{2}+\sigma_{i}\left\|\varphi_{i}\right\|_{c l}^{2},
\end{aligned}
$$

and

1. $J_{1}=f^{\prime}\left(t, s(t), s\left(t-\tau_{1}(t)\right), \int_{t-\sigma_{1}(t)}^{t} s(\xi) d \xi\right) \in R^{n \times n}$ is the Jacobian of $f\left(t, x(t), x\left(t-\tau_{1}(t)\right)\right.$, $\left.\int_{t-\sigma_{1}(t)}^{t} x(s) d s\right)$ at $s(t)$ with the derivative of $f\left(t, x(t), x\left(t-\tau_{1}(t)\right), \int_{t-\sigma_{1}(t)}^{t} x(s) d s\right)$ with respect to $x(t)$,

2. $J_{\tau_{1}}=f^{\prime}\left(t, s(t), s\left(t-\tau_{1}(t)\right), \int_{t-\sigma_{1}(t)}^{t} s(\xi) d \xi\right) \in R^{n \times n}$ is the Jacobian of $f\left(t, x(t), x\left(t-\tau_{1}(t)\right)\right.$, $\left.\int_{t-\sigma_{1}(t)}^{t} x(s) d s\right)$ at $s\left(t-\tau_{1}(t)\right)$ with the derivative of $f\left(t, x(t), x\left(t-\tau_{1}(t)\right), \int_{t-\sigma_{1}(t)}^{t} x(s) d s\right)$ with respect to $x\left(t-\tau_{1}(t)\right)$,

3. $J_{\sigma_{1}}=f^{\prime}\left(t, s(t), s\left(t-\tau_{1}(t)\right), \int_{t-\sigma_{1}(t)}^{t} s(\xi) d \xi\right) \in R^{n \times n}$ is the Jacobian of $f\left(t, x(t), x\left(t-\tau_{1}(t)\right)\right.$, $\left.\int_{t-\sigma_{1}(t)}^{t} x(s) d s\right)$ at $\int_{t-\sigma_{1}(t)}^{t} s(\xi) d \xi$ with the derivative of $f\left(t, x(t), x\left(t-\tau_{1}(t)\right), \int_{t-\sigma_{1}(t)}^{t} x(s) d s\right)$ with respect to $\int_{t-\sigma_{1}(t)}^{t} x(s) d s$.

Theorem 3.1. Consider the close-loop error dynamical network (2.5) with the quadratic cost function (2.6), $\delta>0$, $\mathrm{Q}_{1 i}>0$, and $\mathrm{Q}_{2 i}>0, i=1,2, \ldots, \mathrm{N}$. If there exist symmetric positive definite matrices $\mathrm{P}_{i}, \mathrm{R}_{i}, \mathrm{~S}_{i}, \mathrm{U}_{i}, \mathrm{~V}_{i}, \mathrm{~T}_{i}, \mathrm{~W}_{i}$ and matrices $Z_{i}, i=1,2, \ldots, N$ with appropriately dimensions and positive scalars $\varepsilon_{j}, j=1,2, \ldots, 8$, such that the following LMIs hold:

$$
\begin{aligned}
& \Pi_{1 i}=\left[\begin{array}{ccccccccc}
\Pi_{i 11} & Z_{i}^{\top} & k_{3}^{\top} Z_{i}^{\top} & P_{i} J_{\tau_{1}}^{\top} & P_{i} J_{\sigma_{1}}^{\top} & \rho_{1} N_{1} P_{i} G_{1}^{\top} & \rho_{1} N P_{i} E_{1}^{\top} & P_{i} Q_{1 i} & Z_{i}^{\top} Q_{2 i} \\
* & -U_{i} & 0 & 0 & 0 & 0 & 0 & 0 & 0 \\
* & * & -k_{3}^{2} W_{i} & 0 & 0 & 0 & 0 & 0 & 0 \\
* & * & * & -\varepsilon_{1} \mathrm{I} & 0 & 0 & 0 & 0 & 0 \\
* & * & * & * & -\varepsilon_{2} \mathrm{I} & 0 & 0 & 0 & 0 \\
* & * & * & * & * & -\rho_{1} \varepsilon_{3} \mathrm{NI} & 0 & 0 & 0 \\
* & * & * & * & * & * & -\rho_{1} \varepsilon_{6} \mathrm{NI} & 0 & 0 \\
* & * & * & * & * & * & * & -\mathrm{Q}_{1 i} & 0 \\
* & * & * & * & * & * & * & * & -\mathrm{Q}_{2 i}
\end{array}\right]<0, \\
& \Pi_{2 i}=\left[\begin{array}{ccc}
-e^{-2 \delta \tau_{2}}\left(1-\beta_{2}\right) S_{i} & \rho_{2} N P_{i} G_{2}^{\top} & \rho_{2} N P_{i} E_{2}^{\top} \\
* & -\rho_{2} \varepsilon_{4} N I & 0 \\
* & * & -\rho_{2} \varepsilon_{7} N I
\end{array}\right]<0,
\end{aligned}
$$




$$
\Pi_{2 i}=\left[\begin{array}{ccc}
-e^{-2 \delta \sigma_{2}} T_{i} & \rho_{3} N P_{i} G_{3}^{\top} & \rho_{3} N P_{i} E_{3}^{\top} \\
* & -\rho_{3} \varepsilon_{5} N I & 0 \\
* & * & -\rho_{3} \varepsilon_{8} N I
\end{array}\right]<0,
$$

where

$$
\begin{aligned}
\Pi_{i 11}= & {\left[\begin{array}{ccc}
\tilde{\Gamma}_{i 11} & 0 & 0 \\
* & \Gamma_{i 22} & 0 \\
* & * & \Gamma_{i 33}
\end{array}\right], } \\
\tilde{\Gamma}_{i 11}= & P_{i}\left(J_{1}^{\top}+\delta I\right)+\left(J_{1}+\delta I\right) P_{i}-B_{1 i} Z_{i}-Z_{i}^{\top} B_{1 i}^{\top}+R_{i}+S_{i}+\sigma_{1}^{2} V_{i}+\sigma_{2}^{2} T_{i}+\rho_{1} \varepsilon_{3}\left(\sum_{j=1}^{N}\left(w_{i j}^{1}\right)^{2}\right) I \\
& +\rho_{2} \varepsilon_{4}\left(\sum_{j=1}^{N}\left(w_{i j}^{2}\right)^{2}\right) I+\rho_{3} \varepsilon_{5}\left(\sum_{j=1}^{N}\left(w_{i j}^{3}\right)^{2}\right) I+\frac{e^{2 \delta \tau_{3}}}{1-\beta_{3}} B_{2 i}^{T} u_{i} B_{2 i}+e^{2 \delta \sigma_{3}} B_{3 i}^{\top} W_{i} B_{3 i} \\
& +\rho_{1} \varepsilon_{6} D_{1}^{\top}\left(\sum_{j=1}^{N}\left(w_{i j}^{1}\right)^{2}\right) D_{1}+\rho_{2} \varepsilon_{7} D_{2}^{T}\left(\sum_{j=1}^{N}\left(w_{i j}^{2}\right)^{2}\right) D_{2}+\rho_{3} \varepsilon_{8} D_{3}^{T}\left(\sum_{j=1}^{N}\left(w_{i j}^{3}\right)^{2}\right) D_{3}, \\
\Gamma_{i 22}= & -e^{-2 \delta \tau_{1}}\left(1-\beta_{1}\right) R_{i}+\varepsilon_{1} I_{,} \\
\Gamma_{i 33}= & -e^{-2 \delta \sigma_{1}} V_{i}+\varepsilon_{2} I,
\end{aligned}
$$

then the controlled complex dynamical network (2.1) is EFPS with the system (2.3). Moreover, the feedback control is

$$
u_{i}(t)=-Z_{i} P_{i}^{-1} e_{i}(t), \quad i=1,2, \ldots, N, \quad t \geqslant 0,
$$

and the upper bound of the quadratic cost function (2.6) is as follows:

$$
\begin{aligned}
J< & \sum_{i=1}^{N}\left[e_{i}^{\top}(0) P_{i}^{-1} e_{i}(0)+\int_{-\tau_{1}}^{0} e^{2 \delta s} e_{i}^{\top}(s) P_{i}^{-1} R_{i} P_{i}^{-1} e_{i}(s) d s+\int_{-\tau_{2}}^{0} e^{2 \delta s} e_{i}^{\top}(s) P_{i}^{-1} s_{i} P_{i}^{-1} e_{i}(s) d s\right. \\
& +\int_{-\tau_{3}}^{0} e^{2 \delta s} e_{i}^{\top}(s) P_{i}^{-1} Z_{i}^{\top} U_{i}^{-1} Z_{i} P_{i}^{-1} e_{i}(s) d s+\sigma_{1} \int_{-\sigma_{1}}^{0} \int_{s}^{0} e^{2 \delta \theta} e_{i}^{\top}(\theta) P_{i}^{-1} V_{i} P_{i}^{-1} e_{i}(\theta) d \theta d s \\
& \left.+\sigma_{2} \int_{-\sigma_{2}}^{0} \int_{s}^{0} e^{2 \delta \theta} e_{i}^{\top}(\theta) P_{i}^{-1} T_{i} P_{i}^{-1} e_{i}(\theta) d \theta d s+\sigma_{3} \int_{-\sigma_{3}}^{0} \int_{s}^{0} e^{2 \delta \theta} e_{i}^{\top}(\theta) P_{i}^{-1} Z_{i}^{\top} W_{i}^{-1} Z_{i} P_{i}^{-1} e_{i}(\theta) d \theta d s\right] .
\end{aligned}
$$

Proof. Since $f($.$) is continuous differentiable, it is easy to know that the origin of the nonlinear system$ (2.2) is an asymptotically stable equilibrium point if it is an asymptotically stable equilibrium point of the following linear time-varying delays systems

$$
\begin{aligned}
\dot{e}_{i}(t)= & J_{1} e_{i}(t)+J_{\tau_{1}} e_{i}\left(t-\tau_{1}(t)\right)+J_{\sigma_{1}} \int_{t-\sigma_{1}(t)}^{t} e_{i}(s) d s+\rho_{1} \sum_{j=1}^{N} w_{i j}^{1}\left(G_{1}+\Delta G_{1}(t)\right) e_{j}(t) \\
& +\rho_{2} \sum_{j=1}^{N} w_{i j}^{2}\left(G_{2}+\Delta G_{2}(t)\right) e_{j}\left(t-\tau_{2}(t)\right)+\rho_{3} \sum_{j=1}^{N} w_{i j}^{3}\left(G_{3}+\Delta G_{3}(t)\right) \int_{t-\sigma_{2}(t)}^{t} e_{j}(s) d s \\
& +B_{1 i} u_{i}(t)+B_{2 i} u_{i}\left(t-\tau_{3}(t)\right)+B_{3 i} \int_{t-\sigma_{3}(t)}^{t} u_{i}(s) d s i=1, \ldots, N .
\end{aligned}
$$

Let $Y_{i}=P_{i}^{-1}$ and $y_{i}(t)=Y_{i} e_{i}(t)$. Using the feedback control (3.4), we construct the following LyapunovKrasovskii functional candidate:

$$
\mathrm{V}(\mathrm{t}, \mathrm{e}(\mathrm{t}))=\sum_{\mathrm{k}=1}^{7} \mathrm{~V}_{\mathrm{k}}(\mathrm{t})
$$


where

$$
\begin{aligned}
& V_{1}(t)=\sum_{i=1}^{N} e_{i}^{\top}(t) Y_{i} e_{i}(t), \\
& V_{2}(t)=\sum_{i=1}^{N} \int_{t-\tau_{1}(t)}^{t} e^{2 \delta(s-t)} e_{i}^{\top}(s) Y_{i} R_{i} Y_{i} e_{i}(s) d s, \\
& V_{3}(t)=\sum_{i=1}^{N} \int_{t-\tau_{2}(t)}^{t} e^{2 \delta(s-t)} e_{i}^{\top}(s) Y_{i} S_{i} Y_{i} e_{i}(s) d s, \\
& V_{4}(t)=\sum_{i=1}^{N} \int_{t-\tau_{3}(t)}^{t} e^{2 \delta(s-t)} u_{i}^{\top}(s) u_{i}^{-1} u_{i}(s) d s, \\
& V_{5}(t)=\sigma_{1} \sum_{i=1}^{N} \int_{-\sigma_{1}}^{0} \int_{t+s}^{t} e^{2 \delta(\theta-t)} e_{i}^{\top}(\theta) Y_{i} V_{i} Y_{i} e_{i}(\theta) d \theta, d s, \\
& V_{6}(t)=\sigma_{2} \sum_{i=1}^{N} \int_{-\sigma_{2}}^{0} \int_{t+s}^{t} e^{2 \delta(\theta-t)} e_{i}^{\top}(\theta) Y_{i} T_{i} Y_{i} e_{i}(\theta) d \theta d s, \\
& V_{7}(t)=\sigma_{3} \sum_{i=1}^{N} \int_{-\sigma_{3}}^{0} \int_{t+s}^{t} e^{2 \delta(\theta-t)} u_{i}^{\top}(\theta) W_{i}^{-1} u_{i}(\theta) d \theta d s .
\end{aligned}
$$

It is easy to check that

$$
\sum_{i=1}^{N} \eta_{i}\left\|e_{i}(t)\right\|^{2} \leqslant V(t, e(t)), \quad \forall t \geqslant 0 .
$$

Taking the derivative of $\mathrm{V}(\mathrm{t})$ along the trajectories of system (3.6), we have the following:

$$
\begin{aligned}
\dot{V}_{1}(t)= & 2 \sum_{i=1}^{N} y_{i}^{\top}(t)\left[J_{1} e_{i}(t)+J_{\tau_{1}} e_{i}\left(t-\tau_{1}(t)\right)+J_{\sigma_{1}} \int_{t-\sigma_{1}(t)}^{t} e_{i}(s) d s+\rho_{1} \sum_{j=1}^{N} w_{i j}^{1}\left(G_{1}+\Delta G_{1}(t)\right) e_{j}(t)\right. \\
& +\rho_{2} \sum_{j=1}^{N} w_{i j}^{2}\left(G_{2}+\Delta G_{2}(t)\right) e_{j}\left(t-\tau_{2}(t)\right)+\rho_{3} \sum_{j=1}^{N} w_{i j}^{3}\left(G_{3}+\Delta G_{3}(t)\right) \int_{t-\sigma_{2}(t)}^{t} e_{j}(s) d s \\
& \left.+B_{1 i} u_{i}(t)+B_{2 i} u_{i}\left(t-\tau_{3}(t)\right)+B_{3 i} \int_{t-\sigma_{3}(t)}^{t} u_{i}(s) d s\right] \\
= & \sum_{i=1}^{N} y_{i}^{\top}(t)\left[P_{i}\left(J_{1}^{\top}+\delta I\right)+\left(J_{1}+\delta I\right) P_{i}-B_{1 i} Z_{i}-Z_{i}^{\top} B_{1 i}^{\top}\right] y_{i}(t)+2 \sum_{i=1}^{N} y_{i}^{\top}(t) J_{\tau_{1}} P_{i} y_{i}\left(t-\tau_{1}(t)\right) \\
& +2 \sum_{i=1}^{N} y_{i}^{\top}(t) J_{\sigma_{1}} P_{i} \int_{t-\sigma_{1}(t)}^{t} y_{i}(s) d s+2 \rho_{1} \sum_{i=1}^{N} y_{i}^{\top}(t) \sum_{j=1}^{N} w_{i j}^{1}\left(G_{1}+\Delta G_{1}(t)\right) P_{i} y_{j}(t)-2 \delta V_{1}(t) \\
& +2 \rho_{2} \sum_{i=1}^{N} y_{i}^{\top}(t) \sum_{j=1}^{N} w_{i j}^{2}\left(G_{2}+\Delta G_{2}(t)\right) P_{i} y_{j}\left(t-\tau_{2}(t)\right)+2 \sum_{i=1}^{N} y_{i}^{\top}(t) B_{2 i} u_{i}\left(t-\tau_{3}(t)\right) \\
& +\rho_{3} \sum_{i=1}^{N} y_{i}^{\top}(t) \sum_{j=1}^{N} w_{i j}^{3}\left(G_{3}+\Delta G_{3}(t)\right) P_{i} \int_{t-\sigma_{2}(t)}^{t} y_{j}(s) d s+2 \sum_{i=1}^{N} y_{i}^{\top}(t) B_{3 i} \int_{t-\sigma_{3}(t)}^{t} u_{i}(s) d s, \\
\dot{V}_{2}(t) \leqslant & \sum_{i=1}^{N}\left[y_{i}^{\top}(t) R_{i} y_{i}(t)-e^{-2 \delta \tau_{1}}\left(1-\beta_{1}\right) y_{i}^{\top}\left(t-\tau_{1}(t)\right) R_{i} y_{i}\left(t-\tau_{1}(t)\right)\right]-2 \delta V_{2}(t),
\end{aligned}
$$




$$
\begin{aligned}
& \dot{V}_{3}(t) \leqslant \sum_{i=1}^{N}\left[y_{i}^{\top}(t) S_{i} y_{i}(t)-e^{-2 \delta \tau_{2}}\left(1-\beta_{2}\right) y_{i}^{\top}\left(t-\tau_{2}(t)\right) S_{i} y_{i}\left(t-\tau_{2}(t)\right)\right]-2 \delta V_{3}(t), \\
& \dot{V}_{4}(t) \leqslant \sum_{i=1}^{N}\left[y_{i}^{\top}(t) Z_{i}^{\top} u_{i}^{-1} Z_{i} y_{i}(t)-e^{-2 \delta \tau_{3}}\left(1-\beta_{3}\right) u_{i}^{\top}\left(t-\tau_{3}(t)\right) u_{i}^{-1} u_{i}\left(t-\tau_{3}(t)\right)\right]-2 \delta V_{4}(t), \\
& \dot{V}_{5}(t) \leqslant \sum_{i=1}^{N}\left[\sigma_{1}^{2} y_{i}^{\top}(t) V_{i} y_{i}(t)-\sigma_{1} e^{-2 \delta \sigma_{1}} \int_{t-\sigma_{1}}^{t} y_{i}^{\top}(s) V_{i} y_{i}(s) d s\right]-2 \delta V_{5}(t), \\
& \dot{V}_{6}(t) \leqslant \sum_{i=1}^{N}\left[\sigma_{2}^{2} y_{i}^{\top}(t) T_{i} y_{i}(t)-\sigma_{2} e^{-2 \delta \sigma_{2}} \int_{t-\sigma_{2}}^{t} y_{i}^{\top}(s) T_{i} y_{i}(s) d s\right]-2 \delta V_{6}(t), \\
& \dot{V}_{7}(t) \leqslant \sum_{i=1}^{N}\left[\sigma_{3}^{2} u_{i}^{\top}(t) W_{i}^{-1} u_{i}(t)-\sigma_{3} e^{-2 \delta \sigma_{3}} \int_{t-\sigma_{3}}^{t} u_{i}^{\top}(s) W_{i}^{-1} u_{i}(s) d s\right]-2 \delta V_{7}(t) .
\end{aligned}
$$

According to Lemma 2.7 and Lemma 2.8, we have

$$
\begin{aligned}
& 2 \sum_{i=1}^{N} y_{i}^{\top}(t) J_{\tau_{1}} P_{i} y_{i}\left(t-\tau_{1}(t)\right) \leqslant \frac{1}{\varepsilon_{1}} \sum_{i=1}^{N} y_{i}^{\top}(t) P_{i} J_{\tau_{1}}^{\top} J_{\tau_{1}} P_{i} y_{i}(t) \\
& +\varepsilon_{1} \sum_{i=1}^{N} y_{i}^{\top}\left(t-\tau_{1}(t)\right) y_{i}\left(t-\tau_{1}(t)\right) \\
& 2 \sum_{i=1}^{N} y_{i}^{\top}(t) J_{\sigma_{1}} P_{i} \int_{t-\sigma_{1}(t)}^{t} y_{i}(s) d s \leqslant \frac{1}{\varepsilon_{2}} \sum_{i=1}^{N} y_{i}^{\top}(t) P_{i} J_{\sigma_{1}}^{\top} J_{\sigma_{1}} P_{i} y_{i}(t) \\
& +\varepsilon_{2} \sum_{i=1}^{N} \int_{t-\sigma_{1}(t)}^{t} y_{i}^{\top}(s) d s \int_{t-\sigma_{1}(t)}^{t} y_{i}(s) d s, \\
& 2 \sum_{i=1}^{N} y_{i}^{\top}(t) B_{2 i} u_{i}\left(t-\tau_{3}(t)\right) \leqslant \frac{e^{2 \delta \tau_{3}}}{1-\beta_{3}} \sum_{i=1}^{N} y_{i}^{\top}(t) B_{2 i}^{\top} u_{i} B_{2 i} y_{i}(t) \\
& +e^{-2 \delta \tau_{3}}\left(1-\beta_{3}\right) \sum_{i=1}^{N} u_{i}^{\top}\left(t-\tau_{3}(t)\right) \times u_{i}^{-1} u_{i}\left(t-\tau_{3}(t)\right), \\
& 2 \rho_{1} \sum_{i=1}^{N} y_{i}^{\top}(t) \sum_{j=1}^{N} w_{i j}^{1} G_{1} P_{i} y_{j}(t) \leqslant \rho_{1} \sum_{i=1}^{N} \sum_{j=1}^{N}\left[\varepsilon_{3} y_{i}^{\top}(t)\left(w_{i j}^{1}\right)^{2} y_{i}(t)\right. \\
& \left.+\frac{1}{\varepsilon_{3}} y_{j}^{\top}(t) P_{i} G_{1}^{\top} G_{1} P_{i} y_{j}(t)\right] \\
& =\sum_{i=1}^{N} \sum_{j=1}^{N} \rho_{1} \varepsilon_{3}\left(w_{i j}^{1}\right)^{2} y_{i}^{\top}(t) y_{i}(t) \\
& +\sum_{i=1}^{N} \sum_{j=1}^{N} \frac{\rho_{1}}{\varepsilon_{3}} y_{i}^{\top}(t) P_{i} G_{1}^{\top} G_{1} P_{i} y_{i}(t) \\
& =\sum_{i=1}^{N}\left[\rho_{1} \varepsilon_{3}\left(\sum_{j=1}^{N}\left(w_{i j}^{1}\right)^{2}\right) y_{i}^{\top}(t) y_{i}(t)\right. \\
& \left.+\frac{N \rho_{1}}{\varepsilon_{3}} y_{i}^{\top}(t) P_{i} G_{1}^{\top} G_{1} P_{i} y_{i}(t)\right],
\end{aligned}
$$




$$
\begin{aligned}
& 2 \rho_{2} \sum_{i=1}^{N} y_{i}^{\top}(t) \sum_{j=1}^{N} w_{i j}^{2} G_{2} P_{i} y_{j}\left(t-\tau_{2}(t)\right) \leqslant \rho_{2} \sum_{i=1}^{N} \sum_{j=1}^{N}\left[\varepsilon_{4} y_{i}^{\top}(t)\left(w_{i j}^{2}\right)^{2} y_{i}(t)\right. \\
& \left.+\frac{1}{\varepsilon_{4}} y_{j}^{\top}\left(t-\tau_{2}(t)\right) P_{i} G_{2}^{\top} G_{2} P_{i} y_{j}\left(t-\tau_{2}(t)\right)\right] \\
& =\sum_{i=1}^{N}\left[\rho_{2} \varepsilon_{4}\left(\sum_{j=1}^{N}\left(w_{i j}^{2}\right)^{2}\right) y_{i}^{\top}(t) y_{i}(t)\right. \\
& \left.+\frac{N \rho_{2}}{\varepsilon_{4}} y_{i}^{\top}\left(t-\tau_{2}(t)\right) P_{i} G_{2}^{\top} G_{2} P_{i} \times y_{i}\left(t-\tau_{2}(t)\right)\right], \\
& 2 \sum_{i=1}^{N} y_{i}^{\top}(t) B_{3 i} \int_{t-\sigma_{3}(t)}^{t} u_{i}(s) d s \leqslant e^{2 \delta \sigma_{3}} \sum_{i=1}^{N} y_{i}^{\top}(t) B_{3 i}^{\top} W_{i} B_{3 i} y_{i}(t) \\
& +e^{-2 \delta \sigma_{3}} \sum_{i=1}^{N} \int_{t-\sigma_{3}(t)}^{t} u_{i}^{\top}(s) d s W_{i}^{-1} \int_{t-\sigma_{3}(t)}^{t} u_{i}(s) d s, \\
& \leqslant e^{2 \delta \sigma_{3}} \sum_{i=1}^{N} y_{i}^{\top}(t) B_{3 i}^{\top} W_{i} B_{3 i} y_{i}(t) \\
& +\sigma_{3} e^{-2 \delta \sigma_{3}} \sum_{i=1}^{N} \int_{t-\sigma_{3}}^{t} u_{i}^{\top}(s) W_{i}^{-1} u_{i}(s) d s, \\
& -\sigma_{1} e^{-2 \delta \sigma_{1}} \sum_{i=1}^{N} \int_{t-\sigma_{1}}^{t} y_{i}^{\top}(s) V_{i} y_{i}(s) d s \leqslant-\sigma_{1}(t) e^{-2 \delta \sigma_{1}} \sum_{i=1}^{N} \int_{t-\sigma_{1}(t)}^{t} y_{i}^{\top}(s) V_{i} y_{i}(s) d s \\
& \leqslant-e^{-2 \delta \sigma_{1}} \sum_{i=1}^{N} \int_{t-\sigma_{1}(t)}^{t} y_{i}^{\top}(s) d s V_{i} \int_{t-\sigma_{1}(t)}^{t} y_{i}(s) d s, \\
& -\sigma_{2} e^{-2 \delta \sigma_{2}} \sum_{i=1}^{N} \int_{t-\sigma_{2}}^{t} y_{i}^{\top}(s) T_{i} y_{i}(s) d s \leqslant-\sigma_{2}(t) e^{-2 \delta \sigma_{2}} \sum_{i=1}^{N} \int_{t-\sigma_{2}(t)}^{t} y_{i}^{\top}(s) T_{i} y_{i}(s) d s \\
& \leqslant-e^{-2 \delta \sigma_{2}} \sum_{i=1}^{N} \int_{t-\sigma_{2}(t)}^{t} y_{i}^{\top}(s) d s T_{i} \int_{t-\sigma_{2}(t)}^{t} y_{i}(s) d s . \\
& 2 \rho_{3} \sum_{i=1}^{N} y_{i}^{\top}(t) \sum_{j=1}^{N} w_{i j}^{3} G_{3} P_{i} \int_{t-\sigma_{2}(t)}^{t} y_{j}(s) d s \leqslant \rho_{3} \sum_{i=1}^{N} \sum_{j=1}^{N}\left[\varepsilon_{5} y_{i}^{\top}(t)\left(w_{i j}^{3}\right)^{2} y_{i}(t)\right. \\
& \left.+\frac{1}{\varepsilon_{5}} \int_{t-\sigma_{2}(t)}^{t} y_{j}^{\top}(s) d s P_{i} G_{3}^{\top} G_{3} P_{i} \int_{t-\sigma_{2}(t)}^{t} y_{j}(s) d s\right] \\
& =\sum_{i=1}^{N}\left[\rho_{3} \varepsilon_{5}\left(\sum_{j=1}^{N}\left(w_{i j}^{3}\right)^{2}\right) y_{i}^{\top}(t) y_{i}(t)\right. \\
& \left.+\frac{N \rho_{3}}{\varepsilon_{5}} \int_{t-\sigma_{2}(t)}^{t} y_{i}^{\top}(s) d s P_{i} G_{3}^{\top} G_{3} P_{i} \int_{t-\sigma_{2}(t)}^{t} y_{i}(s) d s\right],
\end{aligned}
$$


According to Assumption 2.6 and Lemma 2.7, we have

$$
\begin{aligned}
2 \rho_{1} \sum_{i=1}^{N} y_{i}^{\top}(t) \sum_{j=1}^{N} w_{i j}^{1} \Delta G_{1}(t) P_{i} y_{j}(t)= & 2 \rho_{1} \sum_{i=1}^{N} \sum_{j=1}^{N} y_{i}^{\top}(t) w_{i j}^{1} D_{1} F(t) E_{1} P_{i} y_{j}(t) \\
\leqslant & \rho_{1} \sum_{i=1}^{N} \sum_{j=1}^{N}\left[\varepsilon_{6} y_{i}^{\top}(t) D_{1}^{\top}\left(w_{i j}^{1}\right)^{2} D_{1} y_{i}(t)\right. \\
& \left.+\frac{1}{\varepsilon_{6}} y_{j}^{\top}(t) P_{i} E_{1}^{\top} F^{\top}(t) F(t) E_{1} P_{i} y_{j}(t)\right] \\
\leqslant & \sum_{i=1}^{N}\left[\rho_{1} \varepsilon_{6} y_{i}^{\top}(t) D_{1}^{\top}\left(\sum_{j=1}^{N}\left(w_{i j}^{1}\right)^{2}\right) D_{1} y_{i}(t)\right. \\
& \left.+\frac{N \rho_{1}}{\varepsilon_{6}} y_{i}^{\top}(t) P_{i} E_{1}^{\top} E_{1} P_{i} y_{i}(t)\right]
\end{aligned}
$$

$$
\begin{aligned}
2 \rho_{2} \sum_{i=1}^{N} y_{i}^{\top}(t) \sum_{j=1}^{N} w_{i j}^{2} \Delta G_{2}(t) P_{i} y_{j}\left(t-\tau_{2}(t)\right)= & 2 \rho_{2} \sum_{i=1}^{N} \sum_{j=1}^{N} y_{i}^{\top}(t) w_{i j}^{2} D_{2} F(t) E_{2} P_{i} y_{j}\left(t-\tau_{2}(t)\right) \\
\leqslant & \rho_{2} \sum_{i=1}^{N} \sum_{j=1}^{N}\left[\varepsilon_{7} y_{i}^{\top}(t) D_{2}^{\top}\left(w_{i j}^{2}\right)^{2} D_{2} y_{i}(t)\right. \\
& \left.+\frac{1}{\varepsilon_{7}} y_{j}^{\top}\left(t-\tau_{2}(t)\right) P_{i} E_{2}^{\top} F^{\top}(t) F(t) E_{2} P_{i} y_{j}\left(t-\tau_{2}(t)\right)\right] \\
\leqslant & \sum_{i=1}^{N}\left[\rho_{2} \varepsilon_{7} y_{i}^{\top}(t) D_{2}^{\top}\left(\sum_{j=1}^{N}\left(w_{i j}^{2}\right)^{2}\right) D_{2} y_{i}(t)\right. \\
& \left.+\frac{N \rho_{2}}{\varepsilon_{7}} y_{i}^{\top}\left(t-\tau_{2}(t)\right) P_{i} E_{2}^{\top} E_{2} P_{i} y_{i}\left(t-\tau_{2}(t)\right)\right],
\end{aligned}
$$

$$
\begin{aligned}
2 \rho_{3} \sum_{i=1}^{N} y_{i}^{\top}(t) \sum_{j=1}^{N} w_{i j}^{3} \Delta G_{3}(t) P_{i} \int_{t-\sigma_{2}(t)}^{t} y_{j}(s) d s= & 2 \rho_{3} \sum_{i=1}^{N} \sum_{j=1}^{N} y_{i}^{\top}(t) w_{i j}^{3} D_{3} F(t) E_{3} P_{i} \int_{t-\sigma_{2}(t)}^{t} y_{j}(s) d s \\
\leqslant & \rho_{3} \sum_{i=1}^{N} \sum_{j=1}^{N}\left[\varepsilon_{8} y_{i}^{\top}(t) D_{3}^{\top}\left(w_{i j}^{3}\right)^{2} D_{3} y_{i}(t)\right. \\
& \left.+\frac{1}{\varepsilon_{8}} \int_{t-\sigma_{2}(t)}^{t} y_{j}^{\top}(s) d s P_{i} E_{3}^{\top} F^{\top}(t) F(t) E_{3} P_{i} \int_{t-\sigma_{2}(t)}^{t} y_{j}(s) d s\right] \\
\leqslant & \sum_{i=1}^{N}\left[\rho_{3} \varepsilon_{8} y_{i}^{\top}(t) D_{3}^{\top}\left(\sum_{j=1}^{N}\left(w_{i j}^{3}\right)^{2}\right) D_{3} y_{i}(t)\right. \\
& \left.+\frac{N \rho_{3}}{\varepsilon_{8}} \int_{t-\sigma_{2}(t)}^{t} y_{i}^{\top}(s) d s P_{i} E_{3}^{\top} E_{3} P_{i} \int_{t-\sigma_{2}(t)}^{t} y_{i}(s) d s\right] .
\end{aligned}
$$

Hence, according to (3.8)-(3.20), we have

$$
\begin{aligned}
\dot{V}(t, e(t))+2 \delta V(t, e(t)) \leqslant & \sum_{i=1}^{N}\left[y _ { i } ^ { \top } ( t ) \left[P_{i}\left(J_{1}^{\top}+\delta I\right)+\left(J_{1}+\delta I\right) P_{i}-B_{1 i} Z_{i}-Z_{i}^{\top} B_{1 i}^{\top}+R_{i}+S_{i}\right.\right. \\
& +Z_{i}^{\top} u_{i}^{-1} Z_{i}+\sigma_{1}^{2} V_{i}+\sigma_{2}^{2} T_{i}+\sigma_{3}^{2} Z_{i}^{\top} W_{i}^{-1} Z_{i}+\frac{1}{\varepsilon_{1}} P_{i} J_{\tau_{1}}^{\top} J_{\tau_{1}} P_{i}+\frac{1}{\varepsilon_{1}} P_{i} J_{\sigma_{1}}^{\top} J_{\sigma_{1}} P_{i} \\
& +\rho_{1} \varepsilon_{3}\left(\sum_{j=1}^{N}\left(w_{i j}^{1}\right)^{2}\right) I+\rho_{2} \varepsilon_{4}\left(\sum_{j=1}^{N}\left(w_{i j}^{2}\right)^{2}\right) I+\rho_{3} \varepsilon_{5}\left(\sum_{j=1}^{N}\left(w_{i j}^{3}\right)^{2}\right) I
\end{aligned}
$$




$$
\begin{aligned}
& +\frac{e^{2 \delta \tau_{3}}}{1-\beta_{3}} B_{2 i}^{\top} u_{i} B_{2 i}+e^{2 \delta \sigma_{3}} B_{3 i}^{\top} W_{i} B_{3 i}+\rho_{1} \varepsilon_{6} D_{1}^{\top}\left(\sum_{j=1}^{N}\left(w_{i j}^{1}\right)^{2}\right) D_{1} \\
& +\rho_{2} \varepsilon_{7} D_{2}^{\top}\left(\sum_{j=1}^{N}\left(w_{i j}^{2}\right)^{2}\right) D_{2}+\frac{N \rho_{1}}{\varepsilon_{3}} P_{i} G_{1}^{\top} G_{1} P_{i}+\frac{N \rho_{1}}{\varepsilon_{6}} P_{i} E_{1}^{\top} E_{1} P_{i} \\
& \left.+\rho_{3} \varepsilon_{8} D_{3}^{T}\left(\sum_{j=1}^{N}\left(w_{i j}^{3}\right)^{2}\right) D_{3}\right] y_{i}(t) \\
& +y_{i}^{\top}\left(t-\tau_{1}(t)\right)\left[-e^{-2 \delta \tau_{1}}\left(1-\beta_{1}\right) R_{i}+\varepsilon_{1} I\right] y_{i}\left(t-\tau_{1}(t)\right) \\
& +y_{i}^{\top}\left(t-\tau_{2}(t)\right)\left[-e^{-2 \delta \tau_{2}}\left(1-\beta_{2}\right) S_{i}+\frac{N \rho_{2}}{\varepsilon_{4}} P_{i} G_{2}^{\top} G_{2} P_{i}\right. \\
& \left.\left.+\frac{N \rho_{2}}{\varepsilon_{7}} P_{i} E_{2}^{\top} E_{2} P_{i}\right] y_{i}\left(t-\tau_{2}(t)\right)+\frac{N \rho_{3}}{\varepsilon_{8}} P_{i} E_{3}^{T} E_{3} P_{i}\right] \int_{t-\sigma_{2}(t)}^{t} y_{i}(s) d s \\
& +\int_{t-\sigma_{1}(t)}^{t} y_{i}^{\top}(s) d s\left[-e^{-2 \delta} \sigma_{1} V_{i}+\varepsilon_{2} I\right] \int_{t-\sigma_{1}(t)}^{t} y_{i}(s) d s \\
& +\int_{t-\sigma_{2}(t)}^{t} y_{i}^{\top}(s) d s\left[-e^{-2 \delta} \sigma_{2} T_{i}+\frac{N \rho_{3}}{\varepsilon_{5}} P_{i} G_{3}^{\top} G_{3} P_{i}\right. \\
& =\sum_{i=1}^{N}\left[\xi_{i}^{\top}(t) \Gamma_{i} \xi_{i}(t)+y_{i}^{\top}\left(t-\tau_{2}(t)\right) \Phi_{i} y_{i}\left(t-\tau_{2}(t)\right)\right. \\
& \left.+\left(\int_{t-\sigma_{2}(t)}^{t} y_{i}^{\top}(s) d s\right) \Upsilon_{i}\left(\int_{t-\sigma_{2}(t)}^{t} y_{i}(s) d s\right)-e_{i}^{\top}(t) \Psi_{i} e_{i}(t)\right],
\end{aligned}
$$

where

$$
\begin{aligned}
& \Gamma_{\mathrm{i}}=\left[\begin{array}{ccc}
\Gamma_{\mathrm{i} 11} & 0 & 0 \\
* & \Gamma_{\mathrm{i} 22} & 0 \\
* & * & \Gamma_{\mathrm{i} 33}
\end{array}\right] \\
& \Gamma_{i 11}=P_{i}\left(J_{1}^{\top}+\delta I\right)+\left(J_{1}+\delta I\right) P_{i}-B_{1 i} Z_{i}-Z_{i}^{\top} B_{1 i}^{\top}+R_{i}+S_{i}+Z_{i}^{\top} U_{i}^{-1} Z_{i}+\sigma_{1}^{2} V_{i} \\
& +\sigma_{2}^{2} T_{i}+\sigma_{3}^{2} Z_{i}^{\top} W_{i}^{-1} Z_{i}+\frac{1}{\varepsilon_{1}} P_{i} J_{\tau_{1}}^{\top} J_{\tau_{1}} P_{i}+\frac{1}{\varepsilon_{2}} P_{i} J_{\sigma_{1}}^{\top} J_{\sigma_{1}} P_{i}+\rho_{1} \varepsilon_{3}\left(\sum_{j=1}^{N}\left(w_{i j}^{1}\right)^{2}\right) I \\
& +\rho_{2} \varepsilon_{4}\left(\sum_{j=1}^{N}\left(w_{i j}^{2}\right)^{2}\right) I+\rho_{3} \varepsilon_{5}\left(\sum_{j=1}^{N}\left(w_{i j}^{3}\right)^{2}\right) I+\frac{e^{2 \delta \tau_{3}}}{1-\beta_{3}} B_{2 i}^{\top} U_{i} B_{2 i}+e^{2 \delta \sigma_{3}} B_{3 i}^{\top} W_{i} B_{3 i} \\
& +\rho_{1} \varepsilon_{6} D_{1}^{\top}\left(\sum_{j=1}^{N}\left(w_{i j}^{1}\right)^{2}\right) D_{1}+\rho_{2} \varepsilon_{7} D_{2}^{T}\left(\sum_{j=1}^{N}\left(w_{i j}^{2}\right)^{2}\right) D_{2}+\frac{N \rho_{1}}{\varepsilon_{3}} P_{i} G_{1}^{\top} G_{1} P_{i} \\
& +\frac{N \rho_{1}}{\varepsilon_{6}} P_{i} E_{1}^{\top} E_{1} P_{i}+\rho_{3} \varepsilon_{8} D_{3}^{\top}\left(\sum_{j=1}^{N}\left(w_{i j}^{3}\right)^{2}\right) D_{3}+P_{i} Q_{1 i} P_{i}+Z_{i}^{\top} Q_{2 i} Z_{i}, \\
& \Gamma_{i 22}=-e^{-2 \delta \tau_{1}}\left(1-\beta_{1}\right) R_{i}+\varepsilon_{1} I \text {, } \\
& \Gamma_{\mathrm{i} 33}=-e^{-2 \delta \sigma_{1}} \mathrm{~V}_{\mathrm{i}}+\varepsilon_{2} \mathrm{I} \text {, } \\
& \Phi_{i}=-e^{-2 \delta \tau_{2}}\left(1-\beta_{2}\right) S_{i}+\frac{N \rho_{2}}{\varepsilon_{4}} P_{i} G_{2}^{T} G_{2} P_{i}+\frac{N \rho_{2}}{\varepsilon_{7}} P_{i} E_{2}^{T} E_{2} P_{i}, \\
& \Upsilon_{i}=-e^{-2 \delta} \sigma_{2} T_{i}+\frac{N \rho_{3}}{\varepsilon_{5}} P_{i} G_{3}^{\top} G_{3} P_{i}+\frac{N \rho_{3}}{\varepsilon_{8}} P_{i} E_{3}^{\top} E_{3} P_{i}
\end{aligned}
$$




$$
\begin{aligned}
\Psi_{i} & =Q_{1 i}+P_{i}^{-1} Z_{i}^{\top} Q_{2 i} Z_{i} P_{i}^{-1}, \\
\xi_{i}(t) & =\left[y_{i}(t), y_{i}\left(t-\tau_{1}(t)\right), \int_{t-\sigma_{1}(t)}^{t} y_{i}(s) d s\right] .
\end{aligned}
$$

Applying Schur complement lemma and Lemma 2.9, the inequalities $\Gamma_{i}<0, \Phi_{i}<0$, and $\Upsilon_{i}<0$ are equivalent to $\Pi_{1 i}<0, \Pi_{2 i}<0$, and $\Pi_{3 i}<0$, respectively. Thus, from (3.1)-(3.3) and (3.21), we obtain

$$
\dot{V}(t, e(t))+2 \delta V(t, e(t)) \leqslant-\sum_{i=1}^{N} e_{i}^{\top}(t) \Psi_{i} e_{i}(t), \quad \forall t \geqslant 0 .
$$

Since $\Psi_{i}>0, i=1,2, \ldots, N$, we obtain

$$
\dot{V}(t, e(t)) \leqslant-2 \delta V(t, e(t)), \quad \forall t \geqslant 0 .
$$

Integrating both sides of (3.23) from 0 to $t$, we have

$$
V(t, e(t)) \leqslant V(0, e(0)) e^{-2 \delta t}, \quad \forall t \geqslant 0 .
$$

Furthermore, taking condition (3.7) into account, we have

$$
\sum_{i=1}^{N} \eta_{i}\left\|e_{i}(t)\right\|^{2} \leqslant V(t, e(t)) \leqslant V(0, e(0)) e^{-2 \delta t}, \quad \forall t \geqslant 0 .
$$

Because $V(t, e(t))$ is radially unbounded, by the Lyapunov-Krasovskii theorem and the solution $\left\|e_{i}(t, \varphi)\right\|$ of the close-loop error dynamical network (2.5) satisfying

$$
\left\|e_{i}(t, \varphi)\right\| \leqslant \sqrt{\frac{\left.\mathcal{M}_{i}\right)}{\eta_{i}}} e^{-\delta t}, i=1,2, \ldots, N, \quad \forall t \geqslant 0,
$$

it implies that the error complex network (2.5) is exponentially stable under the hybrid controller (2.4). Consequently, the controlled respond complex dynamical network (2.1) is EFPS with the drive system (2.3).

Furthermore, from (3.22) and $V(t, e(t)) \geqslant 0$, we get the following

$$
\dot{V}(t, e(t)) \leqslant-\sum_{i=1}^{N} e_{i}^{\top}(t) \Psi_{i} e_{i}(t), \quad \forall t \geqslant 0 .
$$

Integrating both sides of (3.24) from 0 to $t$, we obtain

$$
\sum_{i=1}^{N} \int_{0}^{t} e_{i}^{\top}(t) \Psi_{i} e_{i}(t) \leqslant V(0, e(0))-V(t, e(t)) \leqslant V(0, e(0)), \quad \forall t \geqslant 0
$$

Due to $V(t, e(t)) \geqslant 0$, we get

$$
\sum_{i=1}^{N} \int_{0}^{t} e_{i}^{T}(t) \Psi_{i} e_{i}(t) \leqslant V(0, e(0)) \quad \forall t \geqslant 0
$$

Given $t \rightarrow \infty$, we obtain

$$
J=\sum_{i=1}^{N} \int_{0}^{\infty} e_{i}^{\top}(t) \Psi_{i} e_{i}(t) \leqslant V(0, e(0))=J^{*}
$$

The proof is completed. 
Remark 3.2. This is the first time for investigating the guaranteed cost control for exponential function projective synchronization for complex dynamical networks with mixed time-varying delays and hybrid uncertainties asymmetric coupling delays. There are many works consider the guaranteed cost control of complete synchronization for complex dynamical networks with or without coupling delays [15, 17, 18, $20]$, where the scaling factor is a constant $(\alpha(t)=1)$. Hence, our results are superior and have greater applicability.

Realistically, we need that the system to have an optimal performance. The following theorem presents a method of designing suitable controllers, they can minimize the upper bound of the quadratic cost function (2.6) for the exponential function projective synchronization for complex dynamical networks $(2.1)$

Theorem 3.3. Consider the close-loop error complex dynamical network (2.5) and the quadratic cost function (2.6), the optimization problem:

$$
\begin{aligned}
\min _{\left(\varepsilon_{1}, \varepsilon_{2}, \varepsilon_{3}, \varepsilon_{4}, \varepsilon_{5}, \varepsilon_{6}, \varepsilon_{7}, \varepsilon_{8}, P_{i}, R_{i}, S_{i}, U_{i}, V_{i}, T_{i}, W_{i}, Z_{i}\right)} & \sum_{i=1}^{N}\left\{\omega_{i}+\operatorname{Tr}\left(M_{1 i}\right)+\operatorname{Tr}\left(M_{2 i}\right)+\operatorname{Tr}\left(M_{3 i}\right)+\operatorname{Tr}\left(H_{1 i}\right)\right. \\
& \left.+\operatorname{Tr}\left(H_{2 i}\right)+\operatorname{Tr}\left(H_{3 i}\right)\right\}
\end{aligned}
$$

subject to

(i) LMIs (3.1)-(3.3),

(ii) $\left[\begin{array}{cc}-\omega_{i} & e_{i}^{\top}(0) \\ * & -P_{i}\end{array}\right]<0$,

(iii) $\left[\begin{array}{cc}-\mathrm{M}_{1 \mathrm{i}} & \mathrm{N}_{1 \mathrm{i}}^{\top} \\ * & -\tilde{\mathrm{R}}_{\mathrm{i}}\end{array}\right]<0$,

(iv) $\left[\begin{array}{cc}-\mathrm{M}_{2 i} & \mathrm{~N}_{2 i}^{\top} \\ * & -\widetilde{S}_{i}\end{array}\right]<0$, (v) $\left[\begin{array}{cc}-M_{3 i} & N_{3 i}^{\top} \\ * & -\tilde{U}_{i}\end{array}\right]<0$,

(vi) $\left[\begin{array}{cc}-\mathrm{H}_{1 i} & \mathrm{~L}_{1 \mathrm{i}}^{\mathrm{T}} \\ * & -\tilde{\mathrm{V}}_{\mathrm{i}}\end{array}\right]<0$,

(vii) $\left[\begin{array}{cc}-\mathrm{H}_{2 i} & \mathrm{~L}_{2 i}^{\mathrm{T}} \\ * & -\tilde{\mathrm{T}}_{i}\end{array}\right]<0$,

(viii) $\left[\begin{array}{cc}-\mathrm{H}_{3 i} & \mathrm{~L}_{3 i}^{\mathrm{T}} \\ * & -\tilde{W}_{i}\end{array}\right]<0$,

such that if a feasible solution set of $\varepsilon_{j},(j=1,2, \ldots, 8), P_{i}, R_{i}, S_{i}, U_{i}, V_{i}, T_{i}, W_{i}, Z_{i},(i=1,2, \ldots, N)$, then the controller (3.4) is an optimal reliable guaranteed cost control law which ensures the minimization of quadratic cost function upper bound given in (3.5) for the exponential function projective synchronization of uncertain complex dynamical network (2.1), where

$$
\begin{array}{ll}
\mathrm{N}_{1 i} \mathrm{~N}_{1 i}^{\top}=\int_{-\tau_{1}}^{0} e^{2 \delta s} e_{i}(s) e_{i}^{\top}(s) d s, & \mathrm{~N}_{2 i} \mathrm{~N}_{2 i}^{\top}=\int_{-\tau_{2}}^{0} e^{2 \delta s} e_{i}(s) e_{i}^{\top}(s) d s, \\
\mathrm{~N}_{3 i} \mathrm{~N}_{3 i}^{\top}=\int_{-\tau_{3}}^{0} e^{2 \delta s} e_{i}(s) e_{i}^{\top}(s) d s, & \mathrm{~L}_{1 i} \mathrm{~L}_{1 i}^{\top}=\sigma_{1} \int_{-\sigma_{1}}^{0} \int_{s}^{0} e^{2 \delta \theta} e_{i}(\theta) e_{i}^{\top}(\theta) d \theta d s, \\
\mathrm{~L}_{2 i} \mathrm{~L}_{2 i}^{\top}=\sigma_{2} \int_{-\sigma_{2}}^{0} \int_{s}^{0} e^{2 \delta \theta} e_{i}(\theta) e_{i}^{\top}(\theta) d \theta d s, & \mathrm{~L}_{3 i} \mathrm{~L}_{3 i}^{\top}=\sigma_{3} \int_{-\sigma_{3}}^{0} \int_{s}^{0} e^{2 \delta \theta} e_{i}(\theta) e_{i}^{\top}(\theta) d \theta d s
\end{array}
$$

and $\operatorname{Tr}($.$) denotes the trace of matrix.$

Proof. Define $\mathrm{P}^{-1} \mathrm{R}_{i} \mathrm{P}^{-1}=\tilde{\mathrm{R}}_{i}^{-1}, \mathrm{P}^{-1} \mathrm{~S}_{i} \mathrm{P}^{-1}=\tilde{\mathrm{S}}_{i}^{-1}, \mathrm{P}^{-1} \mathrm{Z}_{i}^{\top} \mathrm{U}_{i}^{-1} \mathrm{Z}_{i} \mathrm{P}^{-1}=\tilde{\mathrm{U}}_{i}^{-1}, \mathrm{P}^{-1} \mathrm{~V}_{i} \mathrm{P}^{-1}=\tilde{\mathrm{V}}_{i}^{-1}, \mathrm{P}^{-1} \mathrm{~T}_{i} \mathrm{P}^{-1}=$ $\tilde{T}_{i}^{-1}, P^{-1} Z_{i}^{\top} W_{i}^{-1} Z_{i} P^{-1}=\tilde{W}_{i}^{-1}$. First, by Theorem 3.1, the feasibility of LMIs (3.1)-(3.3) knows that the uncertain complex dynamical network (2.1) can realize exponential function projective synchronization. Next, it follows from Lemma 2.9, (ii)-(viii) in Theorem 3.3 are equivalent to the inequalities $e_{i}^{\top}(0) P^{-1} e_{\mathfrak{i}}(0)<$ $\omega_{i}, N_{1 i}^{\top} \tilde{R}_{i}^{-1} N_{1 i}<M_{1 i}, N_{2 i}^{\top} \tilde{S}_{i}^{-1} N_{2 i}<M_{2 i}, N_{3 i}^{\top} \tilde{U}_{i}^{-1} N_{3 i}<M_{3 i}, L_{1 i}^{\top} \tilde{V}_{i}^{-1} L_{1 i}<H_{1 i}, L_{2 i}^{\top} \tilde{T}_{i}^{-1} L_{2 i}<H_{2 i}$, 
$\mathrm{L}_{3 i}^{\top} \tilde{W}_{\mathrm{i}}^{-1} \mathrm{~L}_{3 i}<\mathrm{H}_{3 i}$, respectively. Moreover, note that utilizing the trace operator allows one to compute the following expressions

$$
\begin{aligned}
& \int_{-\tau_{1}}^{0} e^{2 \delta s} e_{i}^{\top}(s) P^{-1} R_{i} P^{-1} e_{i}(s) d s=\int_{-\tau_{1}}^{0} e^{2 \delta s} e_{i}^{\top}(s) \tilde{R}_{i}^{-1} e_{i}(s) d s \\
& =\int_{-\tau_{1}}^{0} \operatorname{Tr}\left(e^{2 \delta s} e_{i}^{\top}(s) \tilde{R}_{i}^{-1} e_{i}(s)\right) d s \\
& =\operatorname{Tr}\left(\mathrm{N}_{1 i} \mathrm{~N}_{1 i}^{\top} \tilde{\mathrm{R}}_{\mathrm{i}}^{-1}\right)=\operatorname{Tr}\left(\mathrm{N}_{1 i}^{\top} \tilde{\mathrm{R}}_{\mathrm{i}}^{-1} \mathrm{~N}_{1 \mathrm{i}}\right) \text {, } \\
& \int_{-\tau_{2}}^{0} e^{2 \delta s} e_{i}^{\top}(s) P^{-1} S_{i} P^{-1} e_{i}(s) d s=\int_{-\tau_{2}}^{0} e^{2 \delta s} e_{i}^{\top}(s) \tilde{S}_{i}^{-1} e_{i}(s) d s \\
& =\int_{-\tau_{2}}^{0} \operatorname{Tr}\left(e^{2 \delta s} e_{i}^{\top}(s) \tilde{S}_{i}^{-1} e_{i}(s)\right) d s \\
& =\operatorname{Tr}\left(\mathrm{N}_{2 i} \mathrm{~N}_{2 i}^{\top} \tilde{\mathrm{S}}_{i}^{-1}\right)=\operatorname{Tr}\left(\mathrm{N}_{2 i}^{\top} \tilde{\mathrm{S}}_{i}^{-1} \mathrm{~N}_{2 i}\right) \text {, } \\
& \int_{-\tau_{3}}^{0} e^{2 \delta s} e_{i}^{\top}(s) P^{-1} Z_{i}^{\top} U_{i}^{-1} Z_{i} P^{-1} e_{i}(s) d s=\int_{-\tau_{2}}^{0} e^{2 \delta s} e_{i}^{\top}(s) \tilde{U}_{i}^{-1} e_{i}(s) d s \\
& =\int_{-\tau_{2}}^{0} \operatorname{Tr}\left(e^{2 \delta s} e_{i}^{\top}(s) \tilde{u}_{i}^{-1} e_{i}(s)\right) d s \\
& =\operatorname{Tr}\left(\mathrm{N}_{3 i} \mathrm{~N}_{3 i}^{\top} \tilde{\mathrm{U}}_{\mathrm{i}}^{-1}\right)=\operatorname{Tr}\left(\mathrm{N}_{3 i}^{\top} \tilde{\mathrm{U}}_{\mathrm{i}}^{-1} \mathrm{~N}_{3 i}\right) \text {, } \\
& \sigma_{1} \int_{-\sigma_{1}}^{0} \int_{s}^{0} e^{2 \delta \theta} e_{i}^{\top}(\theta) P^{-1} V_{i} P^{-1} e_{i}(\theta) d \theta d s=\sigma_{1} \int_{-\sigma_{1}}^{0} \int_{s}^{0} e^{2 \delta \theta} e_{i}^{\top}(\theta) \tilde{V}_{i}^{-1} e_{i}(\theta) d \theta d s \\
& =\sigma_{1} \int_{-\sigma_{1}}^{0} \int_{s}^{0} e^{2 \delta \theta} \operatorname{Tr}\left(e_{i}^{\top}(\theta) \tilde{V}_{i}^{-1} e_{i}^{\top}(\theta)\right) d \theta d s \\
& =\operatorname{Tr}\left(\mathrm{L}_{1 i} \mathrm{~L}_{1 i}^{\top} \tilde{\mathrm{V}}_{i}^{-1}\right)=\operatorname{Tr}\left(\mathrm{L}_{1 i}^{\top} \tilde{\mathrm{V}}_{i}^{-1} \mathrm{~L}_{1 i}\right) \text {, } \\
& \sigma_{2} \int_{-\sigma_{2}}^{0} \int_{s}^{0} e^{2 \delta \theta} e_{i}^{\top}(\theta) P^{-1} T_{i} P^{-1} e_{i}(\theta) d \theta d s=\sigma_{2} \int_{-\sigma_{2}}^{0} \int_{s}^{0} e^{2 \delta \theta} e_{i}^{\top}(\theta) \tilde{T}_{i}^{-1} e_{i}(\theta) d \theta d s \\
& =\sigma_{2} \int_{-\sigma_{2}}^{0} \int_{s}^{0} e^{2 \delta \theta} \operatorname{Tr}\left(e_{i}^{\top}(\theta) \tilde{T}_{i}^{-1} e_{i}^{\top}(\theta)\right) d \theta d s \\
& =\operatorname{Tr}\left(\mathrm{L}_{2 i} \mathrm{~L}_{2 \mathrm{i}}^{\top} \tilde{\mathrm{T}}_{\mathrm{i}}^{-1}\right)=\operatorname{Tr}\left(\mathrm{L}_{2 \mathrm{i}}^{\top} \tilde{\mathrm{T}}_{\mathrm{i}}^{-1} \mathrm{~L}_{2 \mathrm{i}}\right) \text {, } \\
& \sigma_{3} \int_{-\sigma_{3}}^{0} \int_{s}^{0} e^{2 \delta \theta} e_{i}^{\top}(\theta) P^{-1} Z_{i}^{\top} W_{i}^{-1} Z_{i} P^{-1} e_{i}(\theta) d \theta d s=\sigma_{3} \int_{-\sigma_{3}}^{0} \int_{s}^{0} e^{2 \delta \theta} e_{i}^{\top}(\theta) \tilde{W}_{i}^{-1} e_{i}(\theta) d \theta d s \\
& =\sigma_{3} \int_{-\sigma_{3}}^{0} \int_{s}^{0} e^{2 \delta \theta} \operatorname{Tr}\left(e_{i}^{\top}(\theta) \tilde{W}_{i}^{-1} e_{i}^{\top}(\theta)\right) d \theta d s \\
& =\operatorname{Tr}\left(\mathrm{L}_{3 i} \mathrm{~L}_{3 i}^{\top} \tilde{W}_{i}^{-1}\right)=\operatorname{Tr}\left(\mathrm{L}_{3 i}^{\top} \tilde{W}_{i}^{-1} \mathrm{~L}_{3 i}\right) \text {. }
\end{aligned}
$$

Therefore, in view of (3.5) one can easily deduce that the following inequality

$$
\begin{aligned}
J^{*}= & \sum_{i=1}^{N}\left\{e_{i}^{\top}(0) P^{-1} e_{i}(0)+\operatorname{Tr}\left(N_{1 i}^{\top} \tilde{R}_{i}^{-1} N_{1 i}\right)+\operatorname{Tr}\left(N_{2 i}^{\top} \tilde{s}_{i}^{-1} N_{2 i}\right)+\operatorname{Tr}\left(N_{3 i}^{\top} \tilde{u}_{i}^{-1} N_{3 i}\right)\right. \\
& \left.+\operatorname{Tr}\left(L_{1 i}^{\top} \tilde{V}_{i}^{-1} L_{1 i}\right)+\operatorname{Tr}\left(\mathrm{L}_{2 i}^{\top} \tilde{T}_{i}^{-1} \mathrm{~L}_{2 i}\right)+\operatorname{Tr}\left(\mathrm{L}_{3 i}^{\top} \tilde{W}_{i}^{-1} \mathrm{~L}_{3 i}\right)\right\} \\
< & \sum_{i=1}^{N}\left\{\omega_{i}+\operatorname{Tr}\left(M_{1 i}\right)+\operatorname{Tr}\left(M_{2 i}\right)+\operatorname{Tr}\left(M_{3 i}\right)+\operatorname{Tr}\left(\mathrm{H}_{1 i}\right)+\operatorname{Tr}\left(\mathrm{H}_{2 i}\right)+\operatorname{Tr}\left(\mathrm{H}_{3 i}\right)\right\}
\end{aligned}
$$


is always satisfied. This shows that minimizing the right-hand part of (3.26) is equivalent to the minimization of the upper bound of the quadratic cost function (2.6) which is given in (3.5). Hence, the proof is completed.

In addition, when $\rho_{1}=\rho_{3}=0, \rho_{2}=1$ and distributed delays $\sigma_{1}(t)=\sigma_{2}(t)=0$, the network model (2.1) is translated into

$$
\dot{x}_{i}(t)=f\left(x_{i}(t), x_{i}\left(t-\tau_{1}(t)\right)\right)+\sum_{j=1}^{N} w_{i j}^{1}\left(G_{1}+\Delta G_{1}(t)\right) x_{j}\left(t-\tau_{2}(t)\right)+U_{i}(t), \quad i=1, \ldots, N .
$$

The closed-loop system for the dynamical error between the complex dynamical network (3.27) and the synchronization state $(2.3)$ with $\sigma_{1}(t)=0$ can be written as

$$
\begin{aligned}
\dot{e}_{i}(t)= & \dot{x}_{i}(t)-\dot{\alpha}(t) s(t)-\alpha(t) \dot{s}(t) \\
= & f\left(x_{i}(t), x_{i}\left(t-\tau_{1}(t)\right)\right)-\alpha(t) f\left(s(t), s\left(t-\tau_{1}(t)\right)\right)+\sum_{j=1}^{N} w_{i j}^{2}\left(G_{2}+\Delta G_{2}(t)\right) e_{j}\left(t-\tau_{2}(t)\right) \\
& +B_{1 i} u_{i}(t)+B_{2 i} u_{i}\left(t-\tau_{3}(t)\right), \quad i=1, \ldots, N, \\
e_{i}(t)= & \varphi_{i}(t)=\phi_{i}(t)-\alpha(t) w(t), \quad t \in\left[-\tau_{\max }, 0\right], \quad i=1, \ldots, N .
\end{aligned}
$$

According to the proof technique in Theorems 3.1 and 3.3, the guaranteed cost control of exponential function projective synchronization for the uncertain complex dynamical network (3.27) can be easily obtained. Thus, we can get the following result from Theorems 3.1 and 3.3.

Corollary 3.4. Consider the close-loop error dynamical network (3.28) with the quadratic cost function (2.6), $\delta>0$, $\mathrm{Q}_{1 i}>0$, and $\mathrm{Q}_{2 i}>0, i=1,2, \ldots, \mathrm{N}$. If there exist symmetric positive definite matrices $\mathrm{P}_{i}, \mathrm{R}_{i}, \mathrm{~S}_{i}, \mathrm{U}_{i}$ and matrices $Z_{i}, i=1,2, \ldots, N$ with appropriate dimensions and positive scalars $\varepsilon_{j}, j=1,2,3$ such that the following LMIs hold:

$$
\begin{aligned}
& \Xi_{1 i}=\left[\begin{array}{ccccc}
\Xi_{i 11} & \mathrm{Z}_{i}^{\top} & \mathrm{P}_{i} J_{\tau_{1}}^{\top} & \mathrm{P}_{i} \mathrm{Q}_{1 i} & \mathrm{Z}_{i}^{\top} \mathrm{Q}_{2 i} \\
* & -\mathrm{U}_{i} & 0 & 0 & 0 \\
* & * & -\varepsilon_{1} \mathrm{I} & 0 & 0 \\
* & * & * & -\mathrm{Q}_{1 i} & 0 \\
* & * & * & * & -\mathrm{Q}_{2 i}
\end{array}\right]<0, \\
& \Xi_{2 i}=\left[\begin{array}{cccc}
-e^{-2 \delta h_{2}}\left(1-\beta_{2}\right) S_{i} & \mathrm{NP}_{i} \mathrm{G}_{2}^{\top} & \mathrm{NP}_{i} \mathrm{E}_{2}^{\mathrm{T}} \\
& * & -\varepsilon_{2} \mathrm{NI} & 0 \\
* & & * & -\varepsilon_{3} \mathrm{NI}
\end{array}\right]<0,
\end{aligned}
$$

where

$$
\begin{aligned}
\Xi_{i 11}= & {\left[\begin{array}{cc}
\Upsilon_{i 11} & 0 \\
* & \Upsilon_{i 22}
\end{array}\right], } \\
\Upsilon_{i 11}= & P_{i}\left(J_{1}^{\top}+\delta I\right)+\left(J_{1}+\delta I\right) P_{i}-B_{1 i} Z_{i}-Z_{i}^{\top} B_{1 i}^{\top}+R_{i}+S_{i}+\varepsilon_{2}\left(\sum_{j=1}^{N}\left(w_{i j}^{2}\right)^{2}\right) \\
& +\frac{e^{2 \delta h_{3}}}{1-\beta_{3}} B_{2 i}^{\top} U_{i} B_{2 i}+\varepsilon_{3} D_{2}^{T}\left(\sum_{j=1}^{N}\left(w_{i j}^{2}\right)^{2}\right) D_{2}, \\
\Upsilon_{i 22}= & -e^{-2 \delta h_{1}}\left(1-\beta_{1}\right) R_{i}+\varepsilon_{1} I,
\end{aligned}
$$

then the controlled complex dynamical network (3.27) is EFPS with the system (2.3). Moreover, the feedback control 
is

$$
u_{i}(t)=-Z_{i} P_{i}^{-1} e_{i}(t), \quad i=1,2, \ldots, N, \quad t \geqslant 0,
$$

and the upper bound of the quadratic cost function (2.6) is as follows:

$$
\begin{aligned}
J< & \sum_{i=1}^{N}\left[e_{i}^{\top}(0) P_{i}^{-1} e_{i}(0)+\int_{-\tau_{1}}^{0} e^{2 \delta s} e_{i}^{\top}(s) P_{i}^{-1} R_{i} P_{i}^{-1} e_{i}(s) d s+\int_{-\tau_{2}}^{0} e^{2 \delta s} e_{i}^{\top}(s) P_{i}^{-1} S_{i} P_{i}^{-1} e_{i}(s) d s\right. \\
& \left.+\int_{-\tau_{3}}^{0} e^{2 \delta s} e_{i}^{\top}(s) P_{i}^{-1} Z_{i}^{\top} U_{i}^{-1} Z_{i}^{\top} P_{i}^{-1} e_{i}(s) d s\right] .
\end{aligned}
$$

The following corollary presents a method of selecting a controller minimizing the upper bound of the guaranteed cost (3.31).

Corollary 3.5. Consider the close-loop error complex dynamical network (3.28) and the quadratic cost function (2.6), the optimization problem:

$$
\min _{\left(\varepsilon_{1}, \varepsilon_{2}, \varepsilon_{3}, P_{i}, R_{i}, S_{i}, U_{i}\right)} \sum_{i=1}^{N}\left\{\omega_{i}+\operatorname{Tr}\left(M_{1 i}\right)+\operatorname{Tr}\left(M_{2 i}\right)+\operatorname{Tr}\left(M_{3 i}\right)\right.
$$

subject to

(i) LMIs, (3.29)-(3.30),

(ii) $\left[\begin{array}{cc}-\omega_{i} & e_{i}^{\top}(0) \\ * & -P_{i}\end{array}\right]<0$,

(iii) $\left[\begin{array}{cc}-M_{1 i} & N_{1 i}^{T} \\ * & -\tilde{R}_{i}\end{array}\right]<0$,

(iv) $\left[\begin{array}{cc}-M_{2 i} & N_{2 i}^{\top} \\ * & -\widetilde{S}_{i}\end{array}\right]<0$,

(v) $\left[\begin{array}{cc}-M_{3 i} & N_{3 i}^{T} \\ * & -\tilde{U}_{i}\end{array}\right]<0$,

such that if a feasible solution set of $\varepsilon_{j},(j=1,2,3), P_{i}, R_{i}, S_{i}, U_{i},(i=1,2, \ldots, N)$, then the controller (3.4) is an optimal reliable guaranteed cost control law which ensures the minimization of quadratic cost function upper bound given in (3.31) for the exponential function projective synchronization of uncertain complex dynamical network (3.27).

\section{Numerical examples}

In this section, we present two examples to illustrate the effectiveness and the reduced conservatism of our results.

Example 4.1. To show the effectiveness of the proposed control scheme, the perturbed Chua's circuit system with mixed time-varying delays uses uncoupled node in the complex dynamical network (2.1). The perturbed Chua's circuit system with mixed time-varying delays (drive system) is given by [4]

$$
\begin{aligned}
& \dot{s}_{1}(t)=p\left(s_{2}\left(t-\tau_{1}(t)\right)-\frac{1}{7}\left(2 s_{1}^{3}(t)-s_{1}(t)\right)\right), \\
& \dot{s}_{2}(t)=s_{1}(t)-u s_{2}(t)+s_{3}\left(t-\tau_{1}(t)\right), \\
& \dot{s}_{3}(t)=-q s_{2}(t)+r \int_{t-\sigma_{1}(t)}^{t} s_{1}^{2}(\theta) d \theta
\end{aligned}
$$


and we take the system (4.1) as identical nodes of network (response networks), which is given by

$$
\begin{aligned}
\left(\begin{array}{c}
\dot{x}_{i 1}(t) \\
\dot{x}_{i 2}(t) \\
\dot{x}_{i 3}(t)
\end{array}\right)= & \left(\begin{array}{c}
p\left(x_{i 2}\left(t-\tau_{1}(t)\right)-\frac{1}{7}\left(2 x_{i 1}^{3}(t)-x_{i 1}(t)\right)\right) \\
x_{i 1}(t)-u x_{i 2}(t)+x_{i 3}\left(t-\tau_{1}(t)\right) \\
-q x_{i 2}(t)+r \int_{t-\sigma_{1}(t)}^{t} x_{i 1}^{2}(s) d s
\end{array}\right)+\rho_{1} \sum_{j=1}^{N} w_{i j}^{1}\left(G_{1}+\Delta G_{1}(t)\right) x_{j}(t) \\
& +\rho_{2} \sum_{j=1}^{N} w_{i j}^{2}\left(G_{2}+\Delta G_{2}(t)\right) x_{j}\left(t-\tau_{2}(t)\right)+\rho_{3} \sum_{j=1}^{N} w_{i j}^{3}\left(G_{3}+\Delta G_{3}(t)\right) \int_{t-\sigma_{2}(t)}^{t} x_{j}(s) d s \\
& +U_{i}(t), i=1,2, \ldots, N
\end{aligned}
$$

where $p, q, r$ and $u$ are real positive constants. It is well known that the system (4.1) exhibits chaotic behavior with the parameters $p, q, r$, and $u$ chosen as $p=7, q=\frac{100}{7}, r=0.07$, and $u=1.5$, the initial condition function $\phi(t)=[0.65 \cos t, 0.3 \cos t,-0.2 \cos t]^{\top}$, the time-varying delay functions $\tau_{1}(t)=$ $0.4 \sin ^{2} t, \tau_{2}(t)=0.3 \sin ^{2} t, \tau_{2}(t)=0.3 \sin ^{2} t, \sigma_{1}(t)=0.2 \cos ^{2} t, \sigma_{2}(t)=0.2 \cos ^{2} t$ and $\sigma_{3}(t)=0.1 \cos ^{2} t$, and the time-varying scaling function $\alpha(t)=0.5 \sin \left(\frac{2 \pi}{15}\right)$ is shown in Figures 2-4. It is stable at the equilibrium point $s(t)=0, s\left(t-\tau_{1}(t)\right)=0, \int_{t-\sigma_{1}(t)}^{t} s(\theta) d \theta=0$ and Jacobian matrices are

$$
\mathrm{J}_{1}=\left[\begin{array}{ccc}
1 & 0 & 0 \\
1 & -1.5 & 0 \\
0 & -\frac{100}{7} & 0
\end{array}\right], \mathrm{J}_{\tau_{1}}=\left[\begin{array}{lll}
0 & 7 & 0 \\
0 & 0 & 1 \\
0 & 0 & 0
\end{array}\right], \mathrm{J}_{\sigma_{1}}=\left[\begin{array}{lll}
0 & 0 & 0 \\
0 & 0 & 0 \\
0 & 0 & 0
\end{array}\right]
$$

The parameters are selected as follows: the coupling strength $\rho_{1}=0.2, \rho_{2}=0.3, \rho_{3}=0.2$, the innercoupling matrices are

$$
\begin{aligned}
& \mathrm{G}_{1}=\left[\begin{array}{lll}
3 & 0 & 0 \\
0 & 3 & 0 \\
0 & 0 & 3
\end{array}\right], \quad \mathrm{G}_{2}=\left[\begin{array}{lll}
2 & 0 & 0 \\
0 & 2 & 0 \\
0 & 0 & 2
\end{array}\right], \quad \mathrm{G}_{3}=\left[\begin{array}{lll}
1 & 0 & 0 \\
0 & 1 & 0 \\
0 & 0 & 1
\end{array}\right], \\
& \Delta \mathrm{G}_{1}(\mathrm{t})=\left[\begin{array}{ccc}
0.2 & 0.1 & 0 \\
0.2 & 0.4 & 0 \\
-0.1 & 0 & 0.3
\end{array}\right]\left[\begin{array}{ccc}
\sin (\mathrm{t}) & 0 & 0 \\
0 & \sin (\mathrm{t}) & 0 \\
0 & 0 & \cos (\mathrm{t})
\end{array}\right]\left[\begin{array}{ccc}
0.2 & 0 & 0.3 \\
0 & 0.3 & 0.1 \\
0 & 0.4 & 0.3
\end{array}\right] \text {, } \\
& \Delta \mathrm{G}_{2}(\mathrm{t})=\left[\begin{array}{ccc}
0.2 & 0.3 & 0 \\
0 & 0.2 & 0.1 \\
0 & -0.2 & 0.4
\end{array}\right]\left[\begin{array}{ccc}
\sin (\mathrm{t}) & 0 & 0 \\
0 & \sin (\mathrm{t}) & 0 \\
0 & 0 & \cos (\mathrm{t})
\end{array}\right]\left[\begin{array}{ccc}
0.2 & 0.2 & 0 \\
-0.1 & 0.2 & 0 \\
0.3 & 0 & 0.4
\end{array}\right] \text {, } \\
& \Delta \mathrm{G}_{3}(\mathrm{t})=\left[\begin{array}{ccc}
0.3 & 0 & 0.3 \\
0 & 0.1 & -0.2 \\
0.1 & 0 & 0.2
\end{array}\right]\left[\begin{array}{ccc}
\sin (\mathrm{t}) & 0 & 0 \\
0 & \sin (\mathrm{t}) & 0 \\
0 & 0 & \cos (\mathrm{t})
\end{array}\right]\left[\begin{array}{ccc}
0.3 & 0.1 & 0 \\
0 & 0.4 & 0.2 \\
0.1 & 0.2 & 0.1
\end{array}\right] \text {, }
\end{aligned}
$$

and the coupling configuration matrices are given respectively as follows:

$$
\begin{aligned}
A & =\left[\begin{array}{ccccc}
-2 & 0 & 1 & 0 & 1 \\
1 & -2 & 0 & 0 & 1 \\
0 & 1 & -1 & 0 & 0 \\
1 & 0 & 1 & -2 & 0 \\
0 & 0 & 0 & 1 & -1
\end{array}\right], \\
B & =\left[\begin{array}{ccccc}
-2 & 0 & 0 & 1 & 1 \\
1 & -2 & 1 & 0 & 0 \\
0 & 0 & -1 & 0 & 1 \\
0 & 0 & 1 & -1 & 0 \\
0 & 1 & 0 & 1 & -2
\end{array}\right], \quad C=\left[\begin{array}{ccccc}
-1 & 0 & 0 & 1 & 0 \\
1 & -1 & 0 & 0 & 0 \\
0 & 1 & -1 & 0 & 0 \\
0 & 0 & 1 & -2 & 1 \\
1 & 1 & 0 & 0 & -2
\end{array}\right],
\end{aligned}
$$

and the topology structure of complex network is shown in Figure 1. 

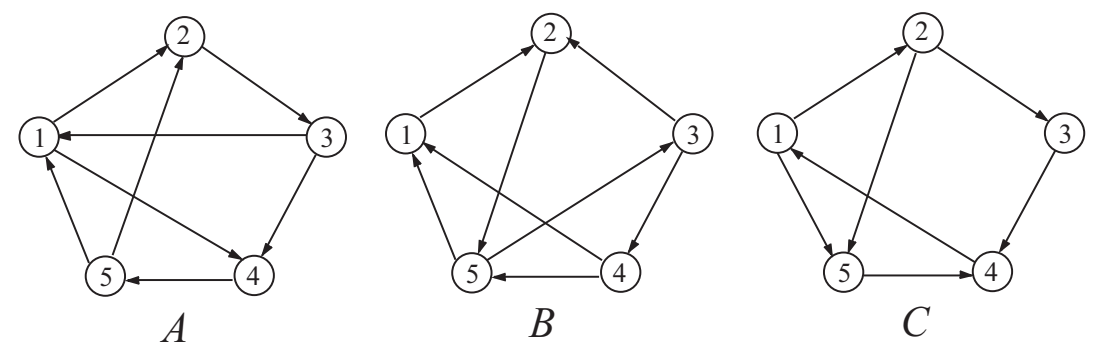

Figure 1: The topology structure of complex network with $\mathrm{N}=5$.

Solution: From the conditions (3.1)-(3.3) of Theorem 3.1 and with positive constants $\delta=0.1, \tau_{1}=0.4$, $\tau_{2}=0.3, \tau_{2}=0.3, \sigma_{1}=0.2, \sigma_{2}=0.2$, and $\sigma_{3}=0.1$, and positive definite matrices

$$
\mathrm{Q}_{1 i}=\left[\begin{array}{lll}
3 & 1 & 1 \\
1 & 4 & 2 \\
1 & 2 & 3
\end{array}\right], \quad \mathrm{Q}_{2 i}=\left[\begin{array}{lll}
3 & 2 & 1 \\
2 & 3 & 1 \\
1 & 1 & 5
\end{array}\right], \quad i=1,2, \ldots, 5,
$$

and

$$
\begin{aligned}
& \mathrm{B}_{11}=\left[\begin{array}{lll}
5 & 0 & 0 \\
0 & 6 & 0 \\
0 & 0 & 4
\end{array}\right], \mathrm{B}_{12}=\left[\begin{array}{lll}
4 & 0 & 0 \\
0 & 4 & 0 \\
0 & 0 & 6
\end{array}\right], \mathrm{B}_{13}=\left[\begin{array}{lll}
5 & 0 & 0 \\
0 & 4 & 0 \\
0 & 0 & 4
\end{array}\right], \\
& \mathrm{B}_{14}=\left[\begin{array}{lll}
5 & 0 & 0 \\
0 & 4 & 0 \\
0 & 0 & 5
\end{array}\right], \mathrm{B}_{15}=\left[\begin{array}{lll}
3 & 0 & 0 \\
0 & 5 & 0 \\
0 & 0 & 4
\end{array}\right], \mathrm{B}_{21}=\left[\begin{array}{ccc}
0.2 & 0 & 0 \\
0 & 0.2 & 0 \\
0 & 0 & 0.5
\end{array}\right], \\
& \mathrm{B}_{22}=\left[\begin{array}{ccc}
0.2 & 0 & 0 \\
0 & 0.5 & 0 \\
0 & 0 & 0.2
\end{array}\right], \mathrm{B}_{23}=\left[\begin{array}{ccc}
0.2 & 0 & 0 \\
0 & 0.2 & 0 \\
0 & 0 & 0.4
\end{array}\right], \mathrm{B}_{24}=\left[\begin{array}{ccc}
0.4 & 0 & 0 \\
0 & 0.3 & 0 \\
0 & 0 & 0.4
\end{array}\right], \\
& \mathrm{B}_{22}=\left[\begin{array}{ccc}
0.2 & 0 & 0 \\
0 & 0.5 & 0 \\
0 & 0 & 0.2
\end{array}\right], \mathrm{B}_{23}=\left[\begin{array}{ccc}
0.2 & 0 & 0 \\
0 & 0.2 & 0 \\
0 & 0 & 0.4
\end{array}\right], \mathrm{B}_{24}=\left[\begin{array}{ccc}
0.4 & 0 & 0 \\
0 & 0.3 & 0 \\
0 & 0 & 0.4
\end{array}\right], \\
& \mathrm{B}_{33}=\left[\begin{array}{ccc}
0.2 & 0 & 0 \\
0 & 0.3 & 0 \\
0 & 0 & 0.4
\end{array}\right], \mathrm{B}_{34}=\left[\begin{array}{ccc}
0.3 & 0 & 0 \\
0 & 0.3 & 0 \\
0 & 0 & 0.4
\end{array}\right], \mathrm{B}_{35}=\left[\begin{array}{ccc}
0.3 & 0 & 0 \\
0 & 0.3 & 0 \\
0 & 0 & 0.2
\end{array}\right],
\end{aligned}
$$

by using the LMI Toolbox in MATLAB, we obtain

$$
\begin{aligned}
& u_{1}(t)=\left[\begin{array}{ccc}
-26.0263 & 12.0950 & -2.8207 \\
6.9120 & -27.5868 & 8.7658 \\
-24.7658 & 36.1151 & -15.6175
\end{array}\right] e_{1}(t), \\
& u_{2}(t)=\left[\begin{array}{ccc}
-97.8263 & 20.2102 & -4.5626 \\
-38.8946 & -10.6816 & 4.8676 \\
-28.3604 & 14.9762 & -10.3270
\end{array}\right] e_{2}(t), \\
& u_{3}(t)=\left[\begin{array}{ccc}
-23.3528 & 4.7142 & -0.8824 \\
8.1784 & -32.7718 & 11.8063 \\
-22.2510 & 27.0087 & -13.2923
\end{array}\right] e_{3}(t), \\
& u_{4}(t)=\left[\begin{array}{ccc}
-38.7719 & 9.3326 & -2.8625 \\
-1.1554 & -25.9686 & 10.4476 \\
-26.4343 & 23.6306 & -13.8490
\end{array}\right] e_{4}(t),
\end{aligned}
$$




$$
u_{5}(t)=\left[\begin{array}{ccc}
-52.4627 & 34.1460 & -8.2239 \\
6.5240 & -29.0009 & 9.6462 \\
-39.6985 & 51.0854 & -19.9977
\end{array}\right] e_{5}(t)
$$

Meanwhile, the optimal upper bound on the guaranteed cost for the exponential function projective synchronization of the complex dynamical networks with mixed time-varying delay and hybrid uncertainties asymmetric coupling delays (4.2) is

$$
\mathrm{J}^{*}=6288.0883 \text {. }
$$

The numerical simulations are carried out using the explicit Runge-Kutta-like method (dde45), interpolation and extrapolation by spline of the third order. Figure 2 shows the chaotic behavior of drive system (4.1) and response network (4.2). Figure 3 shows the function projective synchronization errors between the states of isolate node $\alpha(t) s(t)(4.1)$ and node $x_{i}(t)(4.2)$, where $e_{i j}(t)=x_{i j}(t)-\alpha(t) s_{j}(t)$ for $i=1, \ldots, 5, j=1,2,3$ without hybrid control (2.4). Figure 4 shows the function projective synchronization errors between the states of isolate node $\alpha(t) s(t)$ (4.1) and node $x_{i}(t)$ (4.2) with hybrid control (2.4).

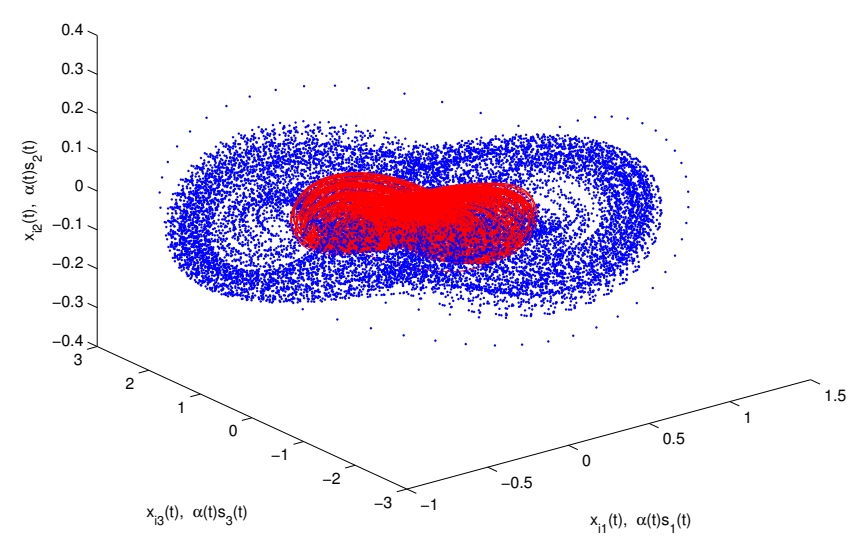

Figure 2: Chaotic behavior of drive system (4.1) and response network (4.2) with the time-varying scaling function $\alpha(t)=$ $0.5 \sin \left(\frac{2 \pi}{15}\right)$.
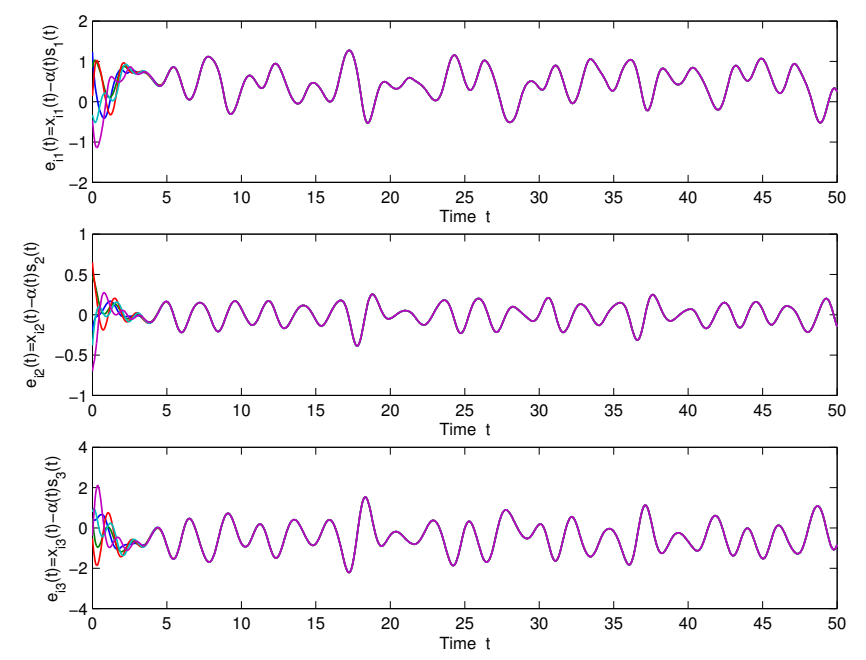

Figure 3: The function projective synchronization errors between the states of isolate node $\alpha(t) s(t)(4.1)$ and node $x_{i}(t)(4.2)$, $i=1,2, \ldots, 5$ without hybrid control (2.4). 

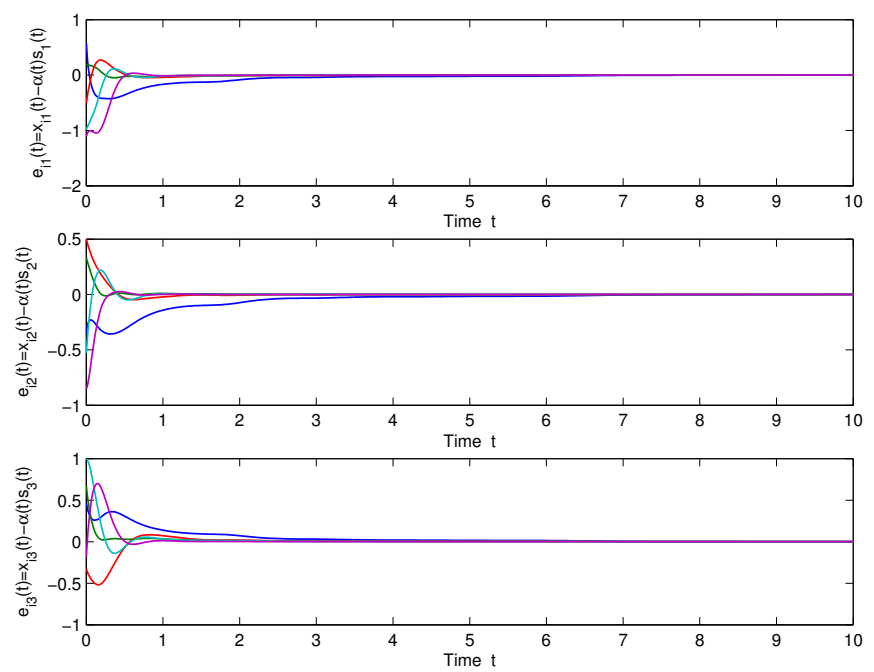

Figure 4: The function projective synchronization errors between the states of isolate node $\alpha(t) s(t)(4.1)$ and node $x_{i}(t)(4.2)$, $i=1,2, \ldots, 5$ with hybrid control (2.4).

Remark 4.2. The advantages of example 4.1 are that the discrete and distributed time-varying delays are different values, i.e., $\tau_{1}(t)=0.4 \sin ^{2} t, \tau_{2}(t)=0.3 \sin ^{2} t, \tau_{2}(t)=0.3 \sin ^{2} t, \sigma_{1}(t)=0.2 \cos ^{2} t, \sigma_{2}(t)=$ $0.2 \cos ^{2} t$ and $\sigma_{3}(t)=0.1 \cos ^{2} t$. Moreover, in these examples we still consider discrete and distributed time-varying delays in the dynamical nodes and the hybrid coupling term simultaneously, hence the synchronization conditions derived in $[15,20]$ cannot be applied to these examples.

Example 4.3. We consider the Chen chaotic system with time delays used as uncoupled node in the network (3.27) (drive system), which is described by [15, 20]

$$
\begin{aligned}
& \dot{s}_{1}(t)=a\left(s_{2}(t)-s_{1}(t)\right) \\
& \dot{s}_{2}(t)=(c-a) s_{1}(t)-s_{1}(t) s_{3}(t)+c s_{2}(t), \\
& \dot{s}_{3}(t)=s_{1}(t) s_{2}(t)-b s_{3}(t)+d\left(s_{3}(t) s_{3}\left(t-\tau_{1}\right),\right.
\end{aligned}
$$

and we take the system (4.3) as identical nodes of network (response networks), which is given by

$$
\begin{aligned}
\left(\begin{array}{c}
\dot{x}_{i 1}(t) \\
\dot{x}_{i 2}(t) \\
\dot{x}_{i 3}(t)
\end{array}\right)= & \left(\begin{array}{c}
a\left(x_{i 2}(t)-x_{i 1}(t)\right) \\
(c-a) x_{i 1}(t)-x_{i 1}(t) x_{i 3}(t)+c x_{i 2}(t) \\
x_{i 1}(t) x_{i 2}(t)-b x_{i 3}(t)+d\left(x_{i 3}(t) x_{i 3}\left(t-\tau_{1}\right)\right)
\end{array}\right)+\sum_{j=1}^{N} w_{i j}^{2}\left(G_{2}+\Delta G_{2}(t)\right) x_{j}\left(t-\tau_{2}\right) \\
& +\mathcal{U}_{i}(t), \quad i=1,2, \ldots, N
\end{aligned}
$$

where $a, b, c$, and $d$ are real positive constants. It is well known that the system (4.3) exhibits chaotic behavior with the parameters $a=35, b=3, c=18$, and $d=3.8$, the initial condition function $\phi(t)=$ $[0.65 \cos t, 0.3 \cos t,-0.2 \cos t]^{\top}$, the time delays $\tau_{1}(t)=0.3, \tau_{2}(t)=0.2$, and the time-varying scaling function $\alpha(t)=0.5+0.2 \sin \left(\frac{2 \pi}{15}\right)$ is shown in Figure 5. It is stable at the equilibrium point $s(t)=0$, $s(t-h(t))=0$ and Jacobian matrices are

$$
J_{1}=\left[\begin{array}{ccc}
-a & a & 0 \\
c-a & c & 0 \\
0 & 0 & -b+d
\end{array}\right], \quad J_{\tau_{1}}=\left[\begin{array}{ccc}
0 & 0 & 0 \\
0 & 0 & 0 \\
0 & 0 & -d
\end{array}\right]
$$

The parameters are selected as follows: $\delta=0.2, \tau_{3}=0.3$, the time-varying scaling function $\alpha(t)=$ 
$0.5+0.2 \sin \left(\frac{2 \pi}{15}\right)$, the inner-coupling matrix are $\mathrm{G}_{2}=\mathrm{D}_{2}=\operatorname{diag}\{1,1,1\}, \mathrm{F}(\mathrm{t})=\operatorname{diag}\{\cos \mathrm{t}, \cos \mathrm{t}, \cos \mathrm{t}\}$, $E_{2}=\operatorname{diag}\{2,2,2\}$, the coupling configuration matrix

$$
W_{2}=\left[\begin{array}{ccccc}
-2 & 1 & 0 & 0 & 1 \\
1 & -3 & 1 & 1 & 0 \\
0 & 1 & -2 & 1 & 0 \\
0 & 1 & 1 & -3 & 1 \\
1 & 0 & 0 & 1 & -2
\end{array}\right]
$$

constant matrices $B_{1 i}=\operatorname{diag}\{5,5,5\}, B_{2 i}=\operatorname{diag}\{0.2,0.2,0.2\}, i=1,2, \ldots, 5$ and select the positive definite matrices $\mathrm{Q}_{1 i}=\mathrm{Q}_{2 i}=\operatorname{diag}\{0.2,0.2,0.2\}, i=1,2, \ldots, 5$. By applying Corollary 3.4 to the complex dynamical network (4.3) and (4.2) and solving the corresponding optimization problem (3.32), the optimal reliable guaranteed cost controllers are given by

$$
\begin{aligned}
& u_{1}(t)=\left[\begin{array}{ccc}
-7.1891 & 2.8784 & 0 \\
0.6014 & -17.5467 & 0 \\
0 & 0 & -11.9823
\end{array}\right] e_{1}(t), \\
& u_{2}(t)=\left[\begin{array}{ccc}
-8.3365 & 2.3016 & 0 \\
0.5560 & -24.6570 & 0 \\
0 & 0 & -17.3618
\end{array}\right] e_{2}(t), \\
& u_{3}(t)=\left[\begin{array}{ccc}
-7.2221 & 2.5598 & 0 \\
0.4079 & -17.0702 & 0 \\
0 & 0 & -11.9264
\end{array}\right] e_{3}(t), \\
& u_{4}(t)=\left[\begin{array}{ccc}
-8.3365 & 2.3016 & 0 \\
0.5560 & -24.6570 & 0 \\
0 & 0 & -17.3618
\end{array}\right] e_{4}(t), \\
& u_{5}(t)=\left[\begin{array}{ccc}
-7.1891 & 2.8784 & 0 \\
0.6014 & -17.5467 & 0 \\
0 & 0 & -11.9823
\end{array}\right] e_{5}(t)
\end{aligned}
$$

and the optimal guaranteed costs of the corresponding closed-loop network are obtained and listed in Table 1. We see that, the optimal upper bound on the guaranteed cost for the synchronization of the complex dynamical network (4.2) obtained from Corollary 3.4 are much less than that obtained in [20]. The results obtained in [20] can not be used for the case when $\delta \neq 0$. Figure 6 shows the function projective synchronization errors between the states of isolate node $\alpha(t) s(t)$ (4.3) and node $x_{i}(t)(4.4)$, where $e_{i j}(t)=x_{i j}(t)-\alpha(t) s_{j}(t)$ for $i=1, \ldots, 5, j=1,2,3$ without hybrid control (2.4). Figure 7 shows the function projective synchronization errors between the states of isolate node $\alpha(t) s(t)$ (4.3) and node $x_{i}(t)$ (4.4) with hybrid control (2.4).

Table 1: The optimal upper bound on the guaranteed cost for the synchronization of the complex dynamical network (4.2) for different values of decay rates $\delta$.

\begin{tabular}{|c|c|c|c|}
\hline decay rates & $\delta=0$ & $\delta=0.5$ & $\delta=1$ \\
\hline$[20]$ & 13,360 & infeasible & infeasible \\
\hline Corollary 3.4 & 4,566 & 6,476 & 8,274 \\
\hline
\end{tabular}




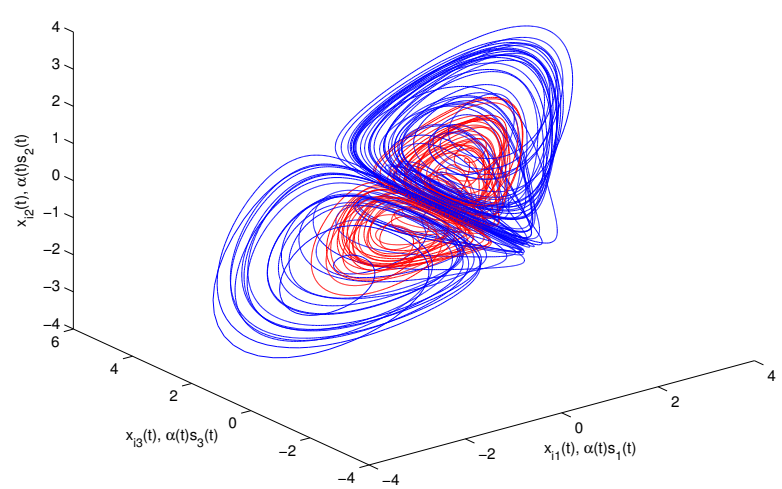

Figure 5: Chaotic behavior of drive system (4.3) and response network (4.4) with the time-varying scaling function $\alpha(t)=$ $0.5+0.2 \sin \left(\frac{2 \pi}{15}\right)$.
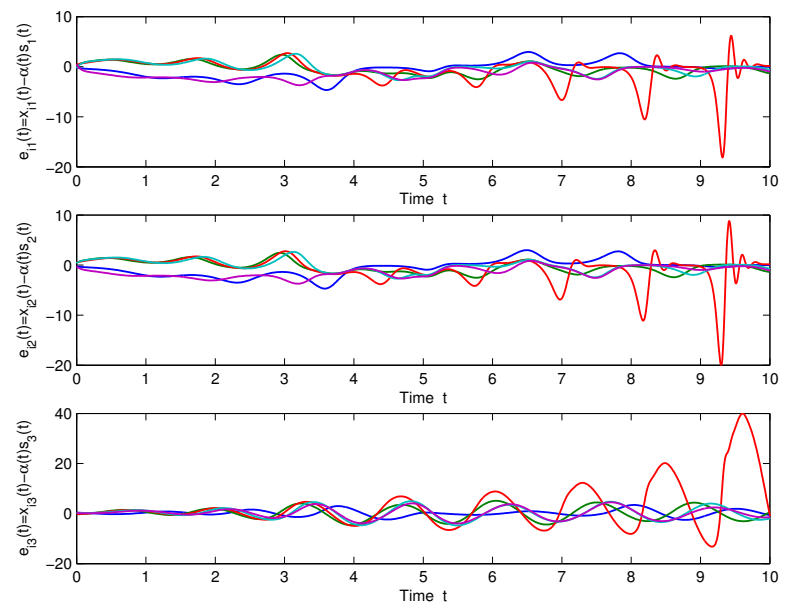

Figure 6: The function projective synchronization errors between the states of isolate node $\alpha(t) s(t)(4.3)$ and node $x_{i}(t)(4.4)$, $i=1,2, \ldots, 5$ without hybrid control (2.4).
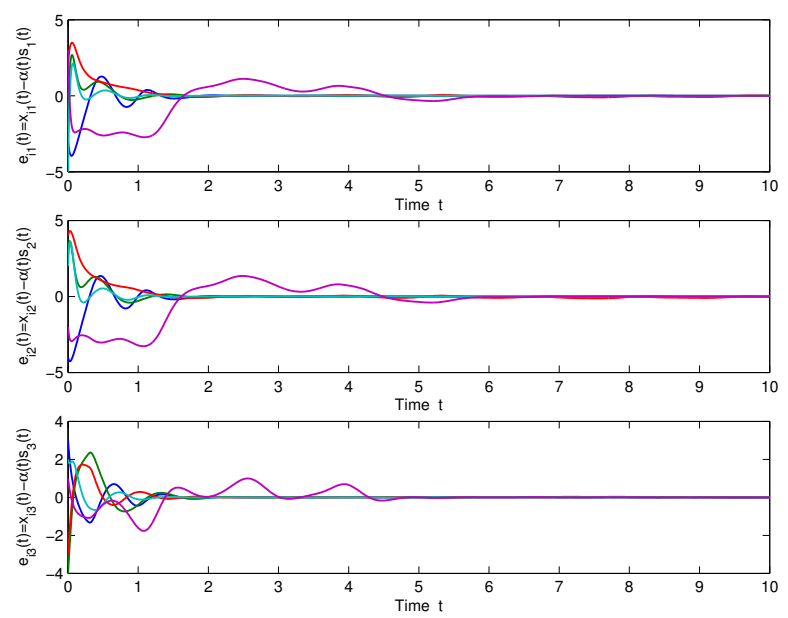

Figure 7: The function projective synchronization errors between the states of isolate node $\alpha(t) s(t)(4.3)$ and node $x_{i}(t)(4.4)$, $i=1,2, \ldots, 5$ with hybrid control (2.4). 


\section{Conclusions}

In this paper, the problem of guaranteed cost control for exponential function projective synchronization (EFPS) for complex dynamical networks with mixed time-varying delays and hybrid uncertainties asymmetric coupling delays is considered. It is not assumed that the uncertainties coupling configuration matrix is symmetric or irreducible. Unlike other works, hybrid control with nonlinear and mixed linear feedback control, which contains error linear term, time-varying delay error linear term and distributed time-varying delay error linear term was considered for guaranteed cost control of EFPS of delayed complex dynamical networks. Based on the construction of novel Lyapunov-Krasovskii functional and linear matrix inequalities, new sufficient conditions are presented for the existence of the optimal guaranteed cost control laws. Moreover, numerical examples are given to demonstrate the effectiveness of proposed guaranteed cost control for EFPS. The results in this article are much less conservative than some existing results in the literature.

\section{Acknowledgment}

The first author was supported by University of Pha Yao. The second author was financially supported by the National Research Council of Thailand and Faculty of Science, Khon Kaen University 2018 and the Thailand Research Fund (TRF), the Office of the Higher Education Commission (OHEC) (grant number: MRG6080239). The third author was financially supported by Chiang Mai University, Chiang Mai, Thailand.

\section{References}

[1] A. Abdurahman, H. Jiang, Z. Teng, Function projective synchronization of impulsive neural networks with mixed timevarying delays, Nonlinear Dynam., 78 (2014), 2627-2638. 1

[2] R. Albert, H. Jeong, A.-L. Barabási, Internet: Diameter of the World-Wide Web, Nature, 401 (1999), 130-131. 1

[3] M. N. Alpaslan Palarkçi, Robust delay-dependent guaranteed cost controller design for uncertain neutral systems, Appl. Math. Comput., 215 (2009), 2936-2949. 1

[4] T. Botmart, P. Niamsup, Exponential synchronization of complex dynamical network with mixed time-varying and hybrid coupling delays via Intermittent control, Adv. Difference Equ., 2014 (2014), 33 pages. 1, 4.1

[5] S. Cai, X. Lei, Z. Liu, Outer synchronization between two hybrid-coupled delayed dynamical networks via aperiodically adaptive intermittent pinning control, Complexity, 21 (2016), 593-605. 1

[6] J. Cao, G. Chen, P. Li, Global synchronization in an array of delayed neural networks with hybrid coupling, IEEE Trans. Syst., Man, Cybern., 38 (2008), 488-498. 1

[7] S. Chang, T. Peng, Adaptive guaranteed cost control of systems with uncertain parameters, IEEE Trans. Automatic Control, 17 (1972), 474-483. 1

[8] W.-H. Chen, Z.-H. Guan, X. Lu, Delay-dependent output feedback guaranteed cost control for uncertain time-delay systems, Automatica J. IFAC, 40 (2004), 1263-1268. 1

[9] B. Cui, X. Lou, Synchronization of chaotic recurrent neural networks with time-varying delays using nonlinear feedback control, Chaos Solitons \& Fractals, 39 (2009), 288-294. 1

[10] H. Du, Function projective synchronization in complex dynamical networks with or without external disturbances via error feedback control, Neurocomputing, 173 (2016), 1443-1449. 1

[11] H. Du, P. Shi, N. Lü, Function projective synchronization in complex dynamical networks with time delay via hybrid feedback control, Nonlinear Anal. Real World Appl., 14 (2013), 1182-1190. 1

[12] M. Faloutsos, P. Faloutsos, C. Faloutsos, On power-law relationships of the Internet topology, Comput. Commun. Rev., 29 (1999), 251-262. 1

[13] K. Gu, V. L. Kharitonov, J. Chen, Stability of time-delay system, Birkhäuser Boston, Boston, (2003). 2.7, 2.8, 2.9

[14] W. He, F. Qian, J. Cao, Pinning-controlled synchronization of delayed neural networks with distributed-delay coupling via impulsive control, Neural Netw., 85 (2017), 1-9. 1

[15] P. He, X.-L. Wang, Y. Li, Guaranteed cost synchronization of complex networks with uncertainties and time-varying delays, complexity, 21 (2015), 381-395. 1, 2.4, 3.2, 4.2, 4.3

[16] H. Jeong, B. Tombor, R. Albert, Z. Oltvai, A.-L. Barabási, The large-scale organization of metabolic network, Nature, 407 (2000), 651-653. 1

[17] T. H. Lee, D. H. Ji, J. H. Park, H. Y. Jung, Decentralized guaranteed cost dynamic control for synchronization of a complex dynamical network with randomly switching topology, Appl. Math. Comput., 219 (2012), 996-1010. 1, 3.2 
[18] T. H. Lee, J. H. Park, D. H. Ji, O. M. Kwon, S. M. Lee, Guaranteed cost synchronization of a complex dynamical network via dynamic feedback control, Appl. Math. Comput., 218 (2012), 6469-6481. 1, 2.4, 3.2

[19] B. Li, Pinning adaptive hybrid synchronization of two general complex dynamical networks with mixed coupling, Appl. Math. Model., 40 (2016), 2983-2998. 1

[20] S. Li, W. Tang, J. Zhang, Guaranteed cost control of synchronisation for uncertain complex delayed networks, Internat. J. Systems Sci., 43 (2012), 566-575. 1, 2.4, 3.2, 4.2, 4.3, 4.3, 1

[21] C.-H. Lien, Delay-dependent and delay-independent guaranteed cost control for uncertain neutral systems with timevarying delays via LMI approach, Chaos, Solitons \& Fractals, 33 (2007), 1017-1027. 1

[22] J. Lu, D. W. C. Ho, J. Cao, Synchronization in an array of nonlinearly coupled chaotic neural networks with delay coupling, Internat. J. Bifur. Chaos Appl. Sci. Engrg., 18 (2008), 3101-3111. 1

[23] Y.-P. Luo, B.-F. Zhou, Guaranteed cost synchronization of complex network systems with delay, Asian J. Control, 17 (2015), 1274-1284. 1

[24] T. Ma, J. Zhang, Y. Zhou, H. Wang, Adaptive hybrid projective synchronization of two coupled fractional-order complex networks with different sizes, Neurocomputing, 164 (2015), 182-189. 1

[25] P. Niamsup, T. Botmart, W. Weera, Modified function projective synchronization of complex dynamical networks with mixed time-varying and asymmetric coupling delays via new hybrid pinning adaptive control, Adv. Difference Equ., 2017 (2017), 31 pages. 1

[26] J. H. Park, K. Choi, Guaranteed cost control of nonlinear neutral systems via memory state feedback, Chaos, Solitons \& Fractals, 24 (2005), 183-190. 1

[27] J. H. Park, O. Kwon, On guaranteed cost control of neutral systems by retarded integral state feedback, Appl. Math. Comput., 165 (2005), 393-404.

[28] G. Rajchakit, Delay-dependent optimal guaranteed cost control of stochastic neural networks with interval nondifferentiable time-varying delays, Adv. Difference Equ., 2013 (2013), 11 pages. 1

[29] R. Rakkiyappan, N. Sakthivel, Cluster synchronization for TS fuzzy complex networks using pinning control with probabilistic time-varying delays, Complexity, 21 (2015), 59-77. 1

[30] K. Sivaranjani, R. Rakkiyappan, Pinning sampled-data synchronization of complex dynamical networks with Markovian jumping and mixed delays using multiple integral approach, Complexity, 21 (2016), 622-632. 1

[31] L. Shi, H. Zhu, S. Zhong, K. Shi, J. Cheng, Function projective synchronization of complex networks with asymmetric coupling via adaptive and pinning feedback control, ISA Trans., 65 (2016), 81-87. 1

[32] L. Shi, H. Zhu, S. Zhong, Y. Zeng, J. Cheng, Synchronization for time-varying complex networks based on control, J. Comput. Appl. Math., 301 (2016), 178-187. 1

[33] Q. Song, J. Cao, On pinning synchronization of directed and undirected complex dynamical networks, IEEE Trans. Circuits Syst. I. Regul. Pap., 57 (2010), 672-680. 1

[34] S. H. Strogatz, Exploring complex networks, Nature, 410 (2001), 268-276. 1

[35] D. Wang, D. Liu, C. Mu, H. Ma, Decentralized guaranteed cost control of interconnected systems with uncertainties: A learning-based optimal control strategy, Neurocomputing, 214 (2016), 297-306. 1

[36] S. Wassrman, K. Faust, Social Network Analysis, Cambridge University Press, Cambridge, (1994). 1

[37] R. J. Williams, N. D. Martinez, Simple rules yield complex food webs, Nature, 404 (2000), 180-183. 1

[38] Y. Wu, C. Li, Y. Wu, J. Kurths, Generalized synchronization between two different complex networks, Commun. Nonlinear Sci. Numer. Simul., 17 (2012), 349-355. 1

[39] Y. Wu, C. Li, A. Yang, L. Song, Y. Wu, Pinning adaptive anti-synchronization between two general complex dynamical networks with non-delayed and delayed coupling, Appl. Math. Comput., 218 (2012), 7445-7452. 1

[40] X. Wu, H. Lu, Generalized projective synchronization between two different general complex dynamical networks with delayed coupling, Phys. Lett. A, 374 (2010), 3932-3941. 1

[41] C. Xie, Y. Xu, D. Tong, Synchronization of time varying delayed complex networks via impulsive control, OptikInternational Journal for Light and Electron Optics, 125 (2014), 3781-3787. 1

[42] Y. Yang, J. Cao, Exponential synchronization of the complex dynamical networks with a coupling delay and impulsive effects, Nonlinear Anal. Real World Appl., 11 (2010), 1650-1659. 1

[43] W. Yu, G. Chen, J. Lü, On pinning synchronization of complex dynamical networks, Automatica, 45 (2009), 429-435. 1

[44] R. Zhang, Y. Yang, Z. Xu, M. Hu, Function projective synchronization in drive-c response dynamical network, Phys. Lett. A, 374 (2010), 3025-3038. 1

[45] Y.-P. Zhao, P. He, H. Saberi Nik, J. Ren, Robust adaptive synchronization of uncertain complex networks with multiple time-varying coupled delays, Complexity, 20 (2015), 49-60. 1 